Accepted Manuscript

Low-Re flow past an isolated cylinder with rounded corners

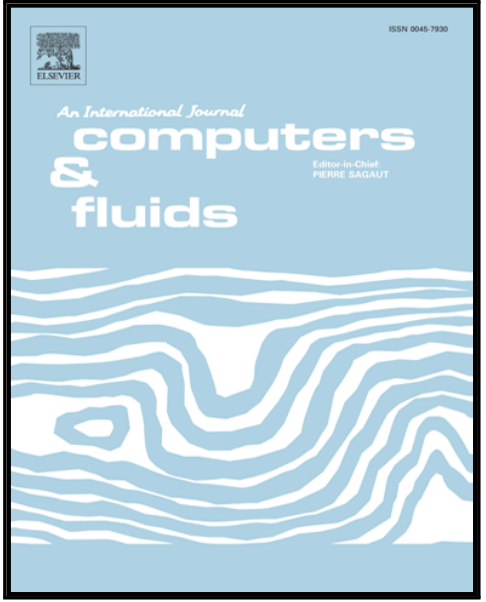

Wei Zhang, Ravi Samtaney

PII:

S0045-7930(16)30210-9

DOI:

10.1016/j.compfluid.2016.06.025

Reference:

CAF 3228

To appear in:

Computers and Fluids

Received date:

15 October 2015

Revised date:

12 June 2016

Accepted date:

28 June 2016

Please cite this article as: Wei Zhang, Ravi Samtaney, Low-Re flow past an isolated cylinder with rounded corners, Computers and Fluids (2016), doi: 10.1016/j.compfluid.2016.06.025

This is a PDF file of an unedited manuscript that has been accepted for publication. As a service to our customers we are providing this early version of the manuscript. The manuscript will undergo copyediting, typesetting, and review of the resulting proof before it is published in its final form. Please note that during the production process errors may be discovered which could affect the content, and all legal disclaimers that apply to the journal pertain. 


\section{Highlights}

- DNS on the separation and transition of flow past a partially rounded cylinder.

- The effect of the corner radius of the cylinder is studied.

- Investigation on time-averaged flow, unsteady flow, turbulent statistics and 3D flow pattern.

- There are drastic variations of the flow pattern between the square cylinder and the partially rounded cylinder, and between the latter and the circular cylinder.

- Non-time-periodicity is significant in the shear layer and at the reattachment point. 


\title{
Low-Re flow past an isolated cylinder with rounded corners
}

\author{
Wei Zhang, Ravi Samtaney
}

Mechanical Engineering, Physical Sciences and Engineering Division, King Abdullah University of Science and Technology, 4700 KAUST, Thuwal 23955-6900, Jeddah, Saudi Arabia

\section{Abstract}

Direct numerical simulation is performed for flow past an isolated cylinder at $R e=$ 1,000. The corners of the cylinder are rounded at different radii, with the non-dimensional radius of curvature varying from $R^{+}=R / D=0.000$ (square cylinder with sharp corners) to 0.500 (circular cylinder), in which $R$ is the corner radius and $D$ is the cylinder diameter. Our objective is to investigate the effect of the rounded corners on the development of the separated and transitional flow past the cylinder in terms of timeaveraged statistics, time-dependent behayior, turbulent statistics and three-dimensional flow patterns. Numerical results reveal that the rounding of the corners significantly reduces the time-averaged drag and the force fluctuations. The wake flow downstream of the square cylinder recovers the slowest and has the largest wake width. However, the statistical quantities do not monotonically vary with the corner radius, but exhibit drastic variations between the cases of square cylinder and partially rounded cylinders, and between the latter and the circular cylinder. The free shear layer separated from the $R^{+}=0.125$ cylinder is the most stable in which the first roll up of the wake vortex occurs furthest from the cylinder and results in the largest recirculation bubble, whose size reduces as $R^{+}$further increases. The coherent and incoherent Reynolds stresses are most pronounced in the near-wake close to the reattachment point, while also being 
noticable in the shear layer for the square and $R^{+}=0.125$ cylinders. The wake vortices translate in the streamwise direction with a convection velocity that is almost constant at approximately $80 \%$ of the incoming flow velocity. These vortices exhibit nearly the same trajectory for the rounded cylinders and are furthest away from the wake centerline for the square one. The flow past the square cylinder is strongly three-dimensional as indicated by the significant primary and secondary enstrophy, while it is dominated by the primary enstrophy $\left(\omega_{z}^{2}\right)$ for the rounded cylinders.

Keywords: Direct numerical simulation, low Reynolds number, turbulent, transition, cylinder, rounded corner

2010 MSC: 76D10, 76F65

\section{Introduction}

The low-Re flow past a cylinder, arguably one of the most classical problem in fluid mechanics, has been extensively studied during the past decades [1]. This canonical flow is of engineering importance due to its relevance in a variety of applications in

5 the fields of aerospace, civil and marine engineering. Most of the earlier studies focus on the flow past a single circular or square cylinder due to their geometrical simplicity and wide applications (e.g., [2-7]), while there are also plenty of numerical and experimental studies on variations such as the inclined cylinder [8-10], the rotating cylinder [11-13], and multiple cylinders in tandem or side-by-side arrangement [14-18]. These 1. studies mainly intended to investigate the effects of the Reynolds number and the geometrical parameters (e.g. the inclination angle or the separation distance between cylinders) on the aerodynamic performance of the cylinder(s) and the near-wake flow patterns. 
The primary difference between flow past a circular and square cylinder is the lo15 cation of flow separation: ( $a$ ) for the circular cylinder, flow separation occurs naturally in response to an adverse pressure gradient (APG) field, and forms a relatively narrow wake; $(b)$ for the square cylinder, the flow is forced to separate at its front corners due to the geometrical discontinuity and reversed flow form on the lateral surfaces, with a relatively wider wake. Previous studies (e.g., $[19,20])$ attribute variations in aerodynamic quantities such as the Strouhal number, lift and drag coefficients and their pulsating amplitudes, and the separation bubble size to geometrieal differences in the low-Re regime. Acknowledging the strong role played by geometry, studies have been undertaken to investigate the effect of the cylinder geometry on the flow dynamics, as seen in Figure 1. The cylinder with sharp corners (square cylinder) is considered as the baseline in which the incoming flow is forced to separate at its front corners. For the cylinder with chamfered corners, consisting of eight flat surfaces, the incoming flow separates at the most upstream corners but relatively smaller recirculation bubbles form on the inclined surfaces with reattachment on the top and bottom surfaces. The flow past the cylinder with rounded corners does not experience forced separation at

so the front corners, and the flow is somewhat similar to that of the circular cylinder. The cylinder with cut corners exhibits two small recirculation bubbles within the corner area, and flow separation on the top and bottom surfaces is reduced in some circumstances.

The cylinder configurations shown in Figure 1 are widely encountered in engineer-

35 ing applications. Here we briefly review a few related studies of low- or high-Re flow past some of such configurations. Larose \& D'Auteuil [21] experimentally studied the 
flow past a chamfered rectangular cylinder at $R e=0.15 \times 10^{6}-4 \times 10^{6}$, with the length (width) of the $45^{\circ}$ chamfer chosen as $4.1 \%$ or $12.5 \%$ of the cylinder diameter. The chamfering of the corners results in a lower drag coefficient. Tamura et al. [22] per-

40 formed numerical simulations on flow past the chamfered and rounded corner cylinders at $R e \sim O\left(10^{4}-10^{6}\right)$. The base pressure coefficient is the smallest for the rounded corner cylinder, and much smaller for the chamfered cylinder than that for the square one. The Strouhal number of the three configurations is within the range 0.10-0.15, with the largest value for the chamfered cylinder and the smallest for the sharp corner cylinder.

45 Tamura \& Miyagi [23] experimentally investigated the turbulent flow past the cylinder at $R e=3 \times 10^{4}$, and found that the chamfering or rounding of the corners promotes the reattachment of the separated shear layer on the lateral surface. This results in a lower drag at all angles of attack (AoA) in the range $-5^{\circ}$ to $30^{\circ}$, and an increased lift. For the cut corner cylinder, Ueda et al. [24] numerically and experimentally studied the effect of the cut corner dimension at $R e \leqslant 10^{4}$. The cut corners alleviate the massive separation and the flow reattaches on the lateral surface, with a consequent narrowing of the width of the shedding vortex street and a strong reduction in drag. The same phenomenon is also observed in the experiment of He et al. [25] wherein a larger size of the cut corner in the streamwise direction produces more effective drag reduction. The virtual aero-shaping effect of the recirculation bubble in the cut corner facilities the flow going through the corner and results in suppression of separation.

Compared with the chamfered and cut corner cylinders, the surface of the rounded corner cylinder is smooth with no curvature discontinuity, and hence the flow on the surface is devoid of any forced separation induced by the abrupt geometry variation. 
Rounded corner cylinders are widely used due to the convenience of manufacturing, and more importantly because of the reduced stress concentration that reduces the potential for structural failure. The radius $R$ of the rounded corner is usually nondimensionalized by the cylinder diameter $D$ as $R^{+}=R / D$. Two extreme geometries are the square cylinder with $R^{+}=0.0$ and the circular cylinder with $R^{+}=0.5$, while a value

$650.0<R^{+}<0.5$ corresponds to a cylinder whose corners are partially rounded but the flat surfaces still remain. Carassale et al. [26] experimentally studied the aerodynamic behavior of the inclined cylinder at $0-45^{\circ}$ angle of attack and $R e=1.7 \times 10^{4}-2.3 \times 10^{5}$ with two rounding radii $R^{+}=1 / 15$ and $2 / 15$. The rounded corner leads to the absence of fixed separation position and promotes flow reattachment on the lateral surface, as 70 those found for the chamfered and cut corner cylinders. Hu et al. [27] experimentally investigated the effect of the corner radius on the near-wake flow at $R e=2,600$ and 6,000 . Their results show that by slightly rounding the corners, the first roll up of the wake vortices occurs further downstream from the cylinder; the vorticity magnitude is significantly attenuated with a value close to that of the circular cylinder. The Strouhal 75 number increases for the rounded cylinder, and both the transverse and streamwise distances of neighboring vortices decrease but their ratio remains almost constant at about 0.29, close to the analytical value of the Kármán street. The near-wake flow is comparably more sensitive to the leading corner radius; the width of the wake is reduced by $25 \%$ as $R^{+}$increases from 0.0 to 0.5 . In the following experimental work by $\mathrm{Hu} \&$ Zhou [28], only two corners of the cylinder are rounded and the cylinder is positioned in various orientations towards the incoming flow at $R e=2,600-8,500$. It is found that as the corner radius increases, the wake recirculation bubble expands but the base 
pressure (i.e., static pressure at the rear stagnation point of the cylinder) rises, resulting in decreased drag, and fluctuating lift and drag forces. The asymmetric cylinder causes the wake centerline to move towards the sharp corner and the shear layer flow is symmetric about the shifted centerline.

Previous studies, some of which were reviewed above, convincingly demonstrate that the aerodynamic loads on the cylinder and the near-wake flow patterns may be significantly altered by the modification of the cylinder geometry. The slight round-

${ }_{90}$ ing or chamfering of the cylinder front corners is very effective in weakening the flow separation on the lateral surfaces of the square cylinder and reducing drag. Since the rounded corner cylinder is widely used in civil and industrial engineering applications, we exclusively focus this type of configuration in this paper and investigate the effect of the rounding of the corners. In addition to the wake flow pattern that has been widely

95 examined in previous studies, the present work highlights detailed investigations on the time-averaged and time-dependent flow patterns, and further provide extensive analysis on the laminar-turbulent transition and three-dimensional flow dynamics. Quantitative data characterizing the flow dynamics are presented and discussed in comparison with previous studies. Our investigation methodology relies on direct numerical simulation (DNS) in which all the flow quantities are properly resolved on a grid. DNS limits our investigations to low- $R e$ flow past the cylinder and presently we fix $R e=1,000$ at which the flow is fully three-dimensionally developed and the free shear layer commences its transition to turbulence [1]. DNS is performed for flow past an isolated cylinder with various rounding corner radii, from $R^{+}=0.000$ (square cylinder with 105 sharp corners) to $R^{+}=0.500$ (circular cylinder), in addition to three intermediate radii 
(a)

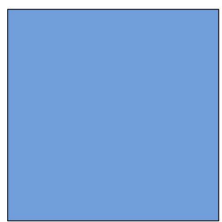

(b)

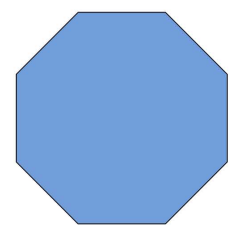

(c)

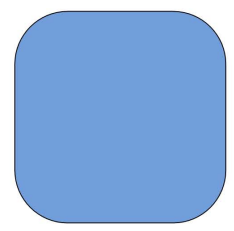

(d)

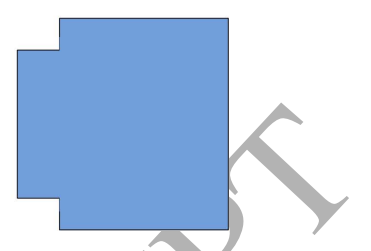

Figure 1: Sectional configurations of the cylinders with various corner geometries: $(a)$ sharp corners; $(b)$ chamfered corners; $(c)$ rounded corners; $(d)$ cut corners.

$R^{+}=0.125,0.250$ and 0.375 chosen to investigate the effect of the rounding radius. The simulation results are analyzed to examine the time-averaged flow field, the unsteady flow behavior, the turbulent statistics and the three-dimensionality of the flow. Aerodynamic quantities on the cylinder are also quantified.

The paper is organized as follows. Section 2 is a general description of the targeted problem, the employed numerical methods, the details of the simulation and the evaluation of simulation quality. Section 3 presents the main simulation results, including the time-averaged and time-dependent behavior, the turbulent statistics and the three-dimensional patterns of the flow. Some conclusions are drawn in section 4.

\section{Numerical setup}

\section{Problem definition}

The present investigation addresses the incoming laminar flow past an isolated cylinder with corners of various radii, as shown in Figure 2. The dimension of the cylinder is $D$ and the four corners are rounded at a radius of curvature $R$. For the threedimensional simulations presented in this paper, the cylinder is assumed straight in the spanwise direction with length $L_{z}$. The flow past the cylinder is modeled in an infinite 

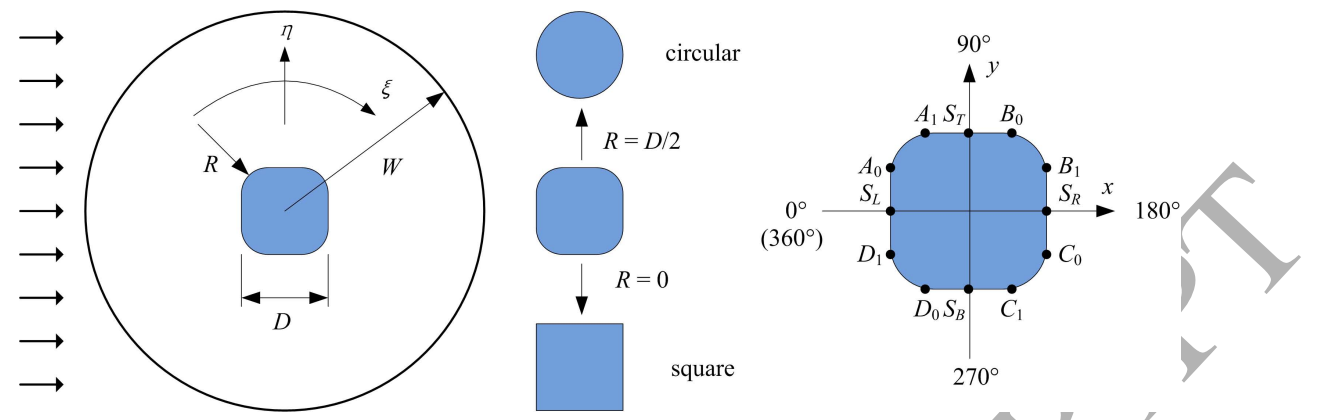

Figure 2: Schematic of the present physical problem and the cylinder configuration. The solid points and notations in the rightmost subfigure denote the reference positions for the demonstration of the simulation results. The reference points $A_{0,1}, B_{0,1}, C_{0,1}$ and $D_{0,1}$ denote the junctions between the flat surface and the curve.

medium, hence the outer boundary is placed sufficiently far away from the cylinder, denoted by $W$ in the figure. Further details on the determination of the various parameters related to the simulation are discussed in section 2.3 .

\subsection{Numerical method}

The computational domain between the cylinder and the outer boundary is discretized by an O-type grid, schematically depicted in Figure 3 for the circular and square cylinder cases. The grid is uniform in the azimuthal direction, but is densely clustered close to and stretched away from the cylinder in the radial direction. A uniform azimuthal grid is employed based on the consideration that the flow pattern on the front side is important for aerodynamic quantities of the cylinder, and that flow separation and transition are critically determined by upstream flow patterns. The size of the first layer grid is fixed at $0.005 \mathrm{D}$ in the wall-normal direction to well resolve the boundary layer, and the stretching ratio is approximately 1.02 for the first 30 layers of 

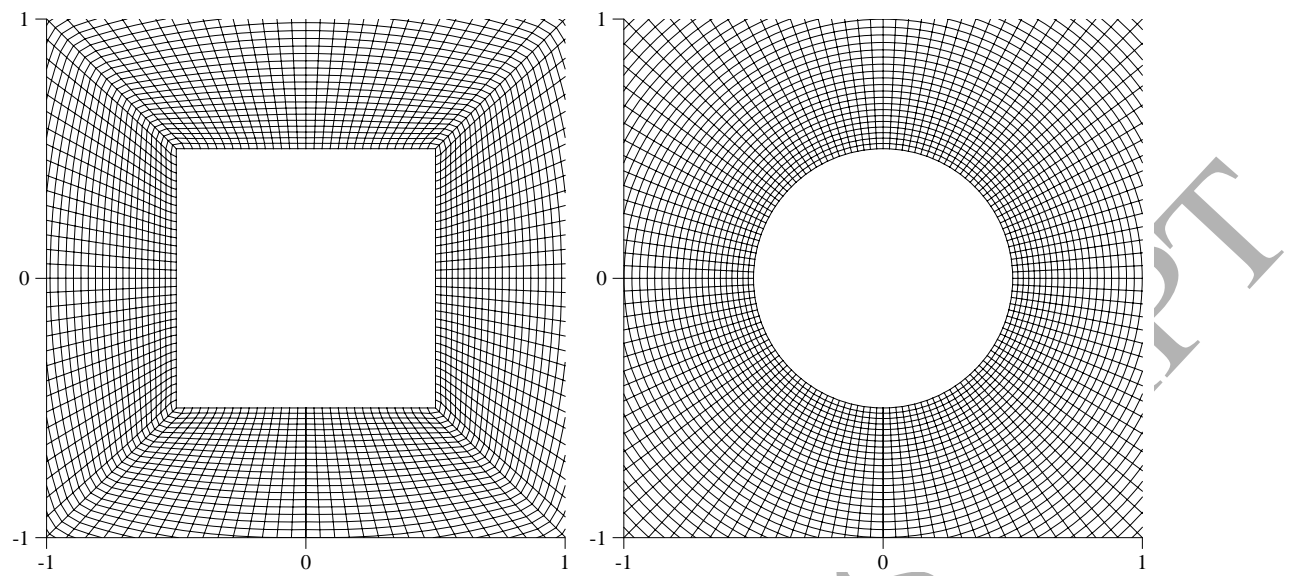

Figure 3: Enlarged view of the O-type grid around the square $\left(R^{+}=0.000\right)$ and circular $\left(R^{+}=0.500\right)$ cylinders. The grid is plotted in every fourth gridline in each direction for clarity.

grid. At the inflow and outflow boundaries, the grid size is $0.5 \mathrm{D}$ in the radial direction and is uniform in the azimuthal direction.

The governing equations for the present simulations are the three-dimensional incompressible Navier-Stokes equations in non-dimensional form:

$$
\begin{gathered}
\frac{\partial u_{k}}{\partial x_{k}}=0 \\
\frac{\partial u_{i}}{\partial t}+\frac{\partial u_{k} u_{i}}{\partial x_{k}}=-\frac{\partial p}{\partial x_{i}}+\frac{1}{\operatorname{Re}} \frac{\partial^{2} u_{i}}{\partial x_{k} \partial x_{k}}
\end{gathered}
$$

where $R e=\rho U_{0} D / \mu$ is the Reynolds number; $\left(u_{1}, u_{2}, u_{3}\right) \equiv(u, v, w)$ are the velocity components; $p$ is the pressure; $\left(x_{1}, x_{2}, x_{3}\right) \equiv(x, y, z)$ are the Cartesian coordinates representing the streamwise, transverse and spanwise directions of the domain. A uniform flow $(u, v, w)=\left(U_{0}, 0,0\right)$ is imposed at the inflow boundary upstream of the cylinder. At the outflow boundary the homogeneous Neumann boundary condition is implemented since the boundary is placed sufficiently far away from the cylinder (see 
section 2.3 for more details). A no-slip and no-penetration boundary condition for velocity is prescribed on the cylinder surface. Periodic boundary condition is employed along the spanwise direction.

The physical problem is numerically simulated by our in-house finite difference code [29-33]. A generalized curvilinear coordinate transformation maps the physical domain $(x, y, z)$ to the computational domain $(\xi, \eta, z)$, in which the governing equations are spatially discretized on a collocated grid. The governing equations are spatially discretized by an energy conservative fourth-order scheme for the convective term [34], and the second-order central difference scheme for the viscous and pressure gradient terms. The discretized governing equations are solved by a semi-implicit fractional step method [35]. The convective and viscous terms are explicitly and implicitly treated, respectively. The modified-Helmholtz equation resulting from the implicit treatment of 155 viscous terms in the predictor step, and the pressure Poisson equation are both solved using a multigrid solver with point- and line-relaxed Gauss-Seidel smoothers. A constant time step size $\Delta t=0.001 D / U_{0}$ is used in all simulations, corresponding to a CFL number smaller than unity. The initial solution to start the three-dimensional simulation is taken as one snapshot from the two-dimensional simulation result, or three-dimensional flow field from another simulation of a case with the next closest corner radius. We first perform the three-dimensional simulation for $300 D / U_{0}$ to wash out the influence of the initial condition, and then starts the production simulation for $500 D / U_{0}$ (about 60-100 shedding cycles depending on the Strouhal number) for data collection and statistics. 
Table 1: Computational parameters in the present study compared with those in the literature. $L_{x}$ and $L_{y}$ are the domain sizes in the cross-sectional plane, with $N_{x}$ and $N_{y}$ the corresponding grid points; $L_{z}$ is the spanwise domain size with $N_{z}$ grid points; $\Delta x_{\min }$ is the minimum wall-normal grid size on the wall; $t_{\text {sim }}$ is the duration of the simulation; $\Delta t$ is the minimum time step size; $T$ is the shedding period. All length scales are normalized by the cylinder diameter $D$. The time is normalized by $D / U_{0}$. See text for more details.

\begin{tabular}{|c|c|c|c|c|c|c|c|c|c|}
\hline Source & Geometry & $\operatorname{Re}$ & $L_{x} \times L_{y}$ & $N_{x} \times N_{y}$ & & $N_{2}$ & $\Delta x_{\min }$ & $t_{\text {sim }}$ & $\Delta t$ \\
\hline Cao et al. [36] & Circular & $60-1,000$ & $30 \times 30$ & & & 40,80 & 0.003 & - & - \\
\hline Labbé \& Wilson [19] & Circular & $100-1,000$ & $15 \times 15$ & & $0.5 \pi-2 \pi$ & $32-128$ & 0.0245 & 300 & 0.005 \\
\hline Lei et al. [37] & Circular & 1,000 & $36 \times 20$ & & $1-6$ & $11-61$ & 0.005 & 400 & 0.005 \\
\hline Persillon \& Braza [38] & Circular & $100-300$ & & & 2.25 & 15 & 0.077 & - & 0.01 \\
\hline Saha et al. [39] & Square & $150-500$ & & $178 \times 80$ & 6 & 22 & - & - & 0.015 \\
\hline Sohankar et al. [20] & Square & $150-50$ & & $209 \times 129$ & 6,10 & $25-41$ & 0.004 & 320 & 0.0125 \\
\hline Sohankar [40] & Square & 000 & 16 & $266 \times 162$ & 4 & 26 & 0.008 & $20 T$ & 0.02 \\
\hline Present & - & & $50 \times 50$ & $512 \times 512$ & 6 & 128 & 0.005 & 500 & 0.001 \\
\hline
\end{tabular}

165 2.3. Computational parameters

The computational parameters used in the present simulations are listed in Table 1 along with certain selected studies for flow past a circular or square cylinder. The key parameters are generally categorized into two groups: the computation domain size $\left(L_{x}, L_{y}\right.$ and $\left.L_{z}\right)$, and the grid resolution and distribution $\left(N_{x}, N_{y}, N_{z}\right.$ and $\left.\Delta x_{\min }\right)$. The time step size is chosen through the CFL condition based on the stability requirement (see section 2.2).

For the low-Re flow past a cylinder presented in this study, the size of the computational domain in the cross-sectional plane affects the simulation results mainly in two aspects: the domain size in the streamwise direction that determines whether the wake 
vortices leave the domain without significant back reflection, and the domain size in the transverse direction that determines whether the blockage introduced by the cylinder notably affects the drag coefficient. For the O-type grid utilized in the simulations, the above effects are only reflected by the parameter $W$ which corresponds to the distance between the cylinder center to the outer boundary. Since a large value $W=50 \mathrm{D}$ i employed, the wake length is $49.5 \mathrm{D}$. Sohankar et al. [8] performed two-dimensional simulation on flow past an inclined rectangular cylinder at $R e \leqslant 200$ with the fully developed outflow boundary condition, similar to the one employed in our investigation, and concluded that a wake length of $26 \mathrm{D}$ is sufficiently large to obtain converged force coefficients. Lei et al. [37] concluded that a wake length of $14.5 \mathrm{D}$ is sufficiently large for the two-dimensional flow past a circular cylinder at $R e=100$. Based on these conclusions and those listed in Table 1 , we believe the wake length $49.5 D$ (i.e., $W=50 D$ ) in our simulation is sufficiently large to obtain reliable simulation results.

The drag coefficient of the cylinder is affected by the blockage ratio in that the momentum of the incoming flow is altered by the presence of the cylinder. Based on the wind tunnel test results, Maskell [41] proposed a correction formula for the timeaveraged drag coefficient as

$$
C_{D, \text { corr }}=\frac{C_{D}}{1+0.96 \varepsilon C_{D}}
$$

For the present blockage ratio $\varepsilon=0.5 D / W=0.01$, the relative difference between the ) corrected and uncorrected coefficients is about $2 \%$ in the worst case, hence $W=50 D$ is considered sufficiently large with respect to the blockage effect.

The spanwise domain size $L_{z}$ for the low-Re flow is crucial since the three-dimensional structures have to be fully developed and well resolved, hence $L_{z}$ should be larger than 
the largest flow structure in this direction. For the square cylinder, Sohankar et al. [20] confirmed that there is no significant difference between the results obtained at $L_{z}=6$ and 10 up to $R e=500$. Labbé \& Wilson [19] found that for $300 \leqslant R e \leqslant 1,000$, a spanwise domain size between $\pi D / 2$ and $\pi D$ could accurately predicts the force coefficients, the Strouhal number and the wavelength of the three-dimensional instability. Persillon \& Braza [38] analyzed the spanwise distance between the kernels of the streamwise vortices and found it is $0.6 D$ at $R e=300$. Since this distance becomes smaller as with increasing Reynolds number, we believe that $L_{z}=6$ is suitable for the present study at $R e=1,000$.

Once the physical domain size is fixed, the resolution and grid distribution are chosen based on the criteria that the boundary layer flow is well resolved and the grid aspect ratio is of an acceptable level. At $R e=1,000$, the maximum size of the first layer grid in the wall-normal direction, as measured in wall units, is about 1.5 , and the maximum grid sizes in the wall-tangent and spanwise directions are about 5.0 and 27.0 (in the worst condition), respectively. It has to be emphasized here that these maximum values correspond to the front stagnation point where the laminar flow impinges on the front surface of the cylinder, while they rapidly decrease as the flow moves along the surface. For the low-Re flow with increasing disorder of fine-scale three-dimensionality [1], the near-wall grid in the present study is sufficiently refined to resolve the boundary layer flow, similar to the turbulent simulations for high-Re flow. The grid resolution is finer in all directions than those used in most studies. 


\subsection{Extracting fluctuations by phase-averaging}

The low-Re flow past a cylinder is dominated by the time-periodic shedding of large-scale vortices, while the turbulent fluctuation and low-frequency modulation (e.g., $[39,42])$ also contribute to the unsteadiness of the flow. Here we decompose the unsteady flow into three parts: the time-averaged component, the time-periodic coherent component and the stochastic incoherent component, as shown by the tripledecomposition relation [43] below:

$$
\phi(\mathbf{x}, t)=\bar{\phi}(\mathbf{x})+\phi^{f}(\mathbf{x}, t)=\bar{\phi}(\mathbf{x})+\phi^{*}(\mathbf{x}, t)+\phi^{\prime}(\mathbf{x}, t)
$$

where $\phi(\mathbf{x}, t)$ is the instantaneous velocity or pressure; $\bar{\phi}(\mathbf{x})$ is the time-averaged component; $\phi^{f}(\mathbf{x}, t)$ is the total fluctuating part; $\phi^{*}(\mathbf{x}, t)$ is the coherent component due to the time-periodic large-scale vortex shedding; $\phi^{\prime}(\mathbf{x}, t)$ is the stochastic fluctuation. Each component on the RHS of Eq.(4) can be computed based on the instantaneous flow field:

$$
\begin{gathered}
\bar{\phi}(\mathbf{x})=\frac{1}{t_{1}-t_{0}} \int_{t_{0}}^{t_{1}} \phi(\mathbf{x}, t) d t, \\
\phi^{f}(\mathbf{x}, t)=\phi(\mathbf{x}, t)-\bar{\phi}(\mathbf{x}), \\
\phi^{*}(\mathbf{x}, t)=\frac{1}{m+n+1} \sum_{i=-m}^{n} \phi(\mathbf{x}, t+i \cdot T)-\bar{\phi}(\mathbf{x}), \\
\phi^{\prime}(\mathbf{x}, t)=\phi(\mathbf{x}, t)-\phi^{*}(\mathbf{x}, t)-\bar{\phi}(\mathbf{x}),
\end{gathered}
$$

where $t_{0}$ and $t_{1}$ are the start and end time of the statistics collection time window; and $T$ is the period of the large-scale vortex shedding. In experiments, the value of $T$ is usually determined from a reference pressure or velocity signal at some fixed point (e.g., $[44,45])$, while in present simulations it is computed and averaged through 
Table 2: Characteristic quantities obtained under various resolutions at $R e=1000$ for the square and circular cylinder cases. $S t$ is the Strouhal number; $C_{D, a v g}$ is the time-averaged drag coefficient; $C_{D, r m s}^{\prime}$ and $C_{L, r m s}^{\prime}$ are the root-mean-square values of the fluctuating drag and lift coefficients; $C_{p b}$ is the base pressure at the rear stagnation point of the cylinder; $\theta_{\text {sep }}$ is the angle of the separation point measured from the front stagnation point; $L_{r}$ is the streamwise length of the recirculation bubble measured from the rear stagnation point.

\begin{tabular}{ccccccccc}
\hline$R^{+}$ & Grid & $S t$ & $C_{D, \text { avg }}$ & $C_{D, r m s}^{\prime}$ & $C_{L, r m s}^{\prime}$ & $-C_{p b}$ & $\theta_{\text {sep }}$ & $L_{r}$ \\
\hline 0.000 & $256 \times 256 \times 128$ & 0.126 & 2.133 & 0.161 & 1.413 & 1.503 & $45.0^{\circ}$ & 0.626 \\
& $384 \times 384 \times 128$ & 0.125 & 2.112 & 0.169 & 1.392 & 1.455 & $45.0^{\circ}$ & 0.601 \\
& $512 \times 512 \times 128$ & 0.122 & 2.089 & 0.178 & 1.378 & 1.416 & $45.0^{\circ}$ & 0.586 \\
& $768 \times 768 \times 192$ & 0.122 & 2.100 & 0.180 & 1.381 & 1.421 & $45.0^{\circ}$ & 0.589 \\
0.500 & $256 \times 256 \times 128$ & 0.219 & 1.062 & 0.035 & 0.159 & 0.898 & $93.3^{\circ}$ & 1.473 \\
& $384 \times 384 \times 128$ & 0.214 & 1.046 & 0.032 & 0.154 & 0.863 & $93.8^{\circ}$ & 1.444 \\
& $512 \times 512 \times 128$ & 0.210 & 1.032 & 0.031 & 0.148 & 0.846 & $94.9^{\circ}$ & 1.424 \\
& $768 \times 768 \times 192$ & 0.211 & 1.040 & 0.031 & 0.147 & 0.844 & $94.7^{\circ}$ & 1.421 \\
\hline
\end{tabular}

spectral analysis on the velocity signal at several probes. Since we mainly focus on the time-periodic low-Re flow, phase jitter (e.g., [46]) is not a major concern and is omitted [47, 48]. The first term on the RHS of Eq.(7) is the phase-averaging over a number of instantaneous flow fields of the same phase. In what follows, we use the overbar $\bar{\phi}$ to denote time-ayeraging during the whole simulation, and the results are spanwise averaged unless specified otherwise.

225 2.5. Grid sensitivity study

A grid sensitivity study is performed to evaluate the effect of the spatial resolution on our simulation results. We simulate the three-dimensional flow past a square or circular cylinder at various resolutions, keeping other computational/geometrical parameters fixed as listed in Table 1. The computed characteristic quantities are listed in Table 2. Generally, consistent predictions are achieved for several quantities under all 
resolutions, and the observed consistency is better for the circular cylinder case. The maximum relative difference between the results obtained under the $384 \times 384 \times 128$ and $512 \times 512 \times 128$ grids is about $5 \%$ and $3 \%$ for the square and circular cylinder cases, respectively. By increasing the spatial resolution, the maximum relative difference between the results obtained under the $512 \times 512 \times 128$ and $768 \times 768 \times 192$ grids decreases to about $2 \%$ and $1 \%$, respectively. We can safely conclude that the $512 \times 512 \times 128$ grid is sufficient to predict the flow characteristics including the fluctuating forces, and will be employed in all following simulations. A posteriori evaluation of the effect of the spatial resolution and grid distribution on the simulation results will be further presented in section 2.7 .

\subsection{Code validation}

In addition to the comparison of geometrical and grid parameters listed in Table 1, various statistical quantities obtained using the $512 \times 512 \times 128$ grid are listed in Table 3 and compared with the previous studies for the square and circular cylinder cases.

Our results are generally consistent with previous studies especially for the Strouhal number and the time-averaged drag coefficient. It is discussed earlier that the simulation results are affected by the temporal and spatial resolutions and the duration of the simulation (see Table 1 for the grid resolution and temporal duration). Certain quantities such as the fluctuating force coefficients and base pressure can vary significantly in . yarious simulations (e.g., see Evangelinos \& Karniadakis [54] and Henderson \& Karniadakis [55]). Supported by the spatial resolution examination in section 2.5 , we believe our simulations provide reliable predictions on the statistical quantities and accurately capture the effect of the rounded corners. More quantitative comparisons are presented 
Table 3: Comparison of several characteristic quantities, at $R e=1000$ except those indicated otherwise.

Refer Table 2 for the physical meanings of the quantities. The superscript asterisk denotes the estimated

value extracted from one or more figures in the corresponding cited paper.

\begin{tabular}{|c|c|c|c|c|c|c|c|c|}
\hline Source & $R^{+}$ & $S t$ & $C_{D, a v g}$ & $C_{D, r m s}^{\prime}$ & $C_{L, r m s}^{\prime}$ & $-C_{p b}$ & $\theta_{\text {sep }}$ & $L_{r}$ \\
\hline Dutta et al. [49] $(\operatorname{Re}=1,340)$ & 0.000 & 0.143 & $2.23^{*}$ & & & & & \\
\hline Norberg [50] & 0.000 & $0.13^{*}$ & & & & & & \\
\hline Okajima [51] & 0.000 & $0.13^{*}$ & & & & & & \\
\hline Okajima et al. [52] & 0.000 & $0.14^{*}$ & $1.9^{*}$ & & & & & \\
\hline Sohankar [40] & 0.000 & $0.12^{*}$ & $2.08^{*}$ & $0.22^{*}$ & $1.45^{*}$ & & & \\
\hline Sohankar [53] & 0.000 & $0.12^{*}$ & $2.06^{*}$ & $0.23^{*}$ & & & & \\
\hline \multirow[t]{5}{*}{ Present } & 0.000 & 0.122 & 2.089 & 0.178 & 1.378 & 1.416 & $45.0^{\circ}$ & 0.586 \\
\hline & 0.125 & 0.152 & 1.168 & & 0.064 & 0.690 & $51.9^{\circ}$ & 3.307 \\
\hline & 0.250 & 0.198 & 1.035 & 0015 & 0.054 & 0.732 & $63.4^{\circ}$ & 2.091 \\
\hline & 0.375 & 0.210 & 0.971 & 0.016 & 0.066 & 0.739 & $79.1^{\circ}$ & 1.825 \\
\hline & 0.500 & 0.210 & 1.032 & 0.031 & 0.148 & 0.846 & $94.9^{\circ}$ & 1.424 \\
\hline Cao et al. [36] & 0.500 & & $1.05^{\circ}$ & & & & $94^{\circ *}$ & \\
\hline Evangelinos \& Karniadakis [54] & 0.500 & 0.202 & 1.019 & 0.035 & 0.099 & 0.843 & & \\
\hline Henderson \& Karniadakis [55] & 0.500 & 0.211 & 1.200 & 0.049 & 0.205 & 1.123 & & \\
\hline Norberg [56] & 0.500 & $0.21^{*}$ & & & & $0.8^{*}$ & & \\
\hline Norberg [57] & & 0.210 & & & $0.1^{*}$ & & & \\
\hline Labbé \& Wilson [19] & 0.500 & 0.215 & 1.200 & & & & & \\
\hline Lei et al. [37] & 0.500 & 0.20 & 1.11 & & $0.33^{*}$ & 1.0 & $97.9^{\circ}$ & $1.15^{*}$ \\
\hline Papaioannou et al. [14] & 0.500 & 0.216 & 1.030 & & & $0.82^{*}$ & & \\
\hline
\end{tabular}

in the following sections which further ensure the reliability of our code.

255 2.7. Assessment of the simulation quality

Although the spatial and temporal resolutions of our DNS are better than a number selected studies, it is still necessary to do a posteriori evaluation of the simulation quality to guarantee that the small-scale turbulent structures are well resolved. Figure 4 shows the size of the grid adjacent to the surface for the square $\left(R^{+}=0.000\right)$ and 260 circular $\left(R^{+}=0.500\right)$ cylinders. The profiles for the other three cases are similar to 

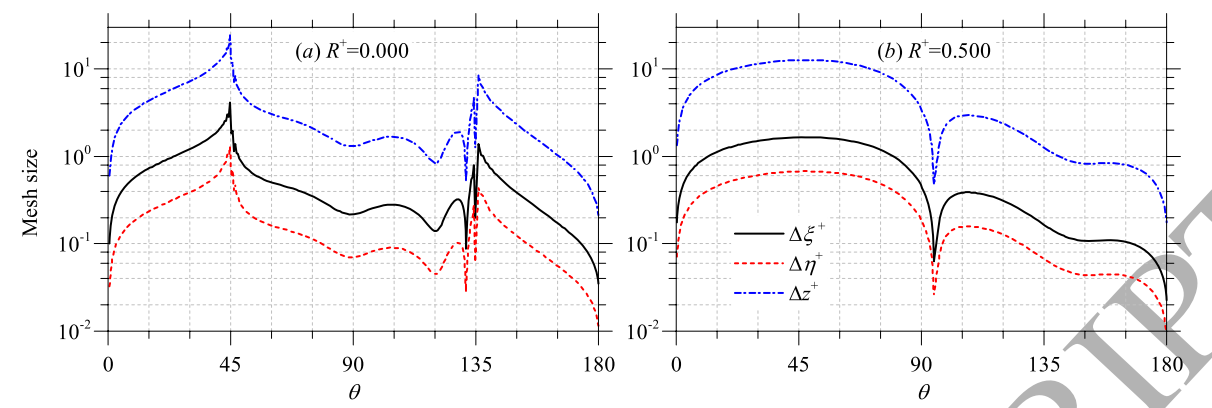

Figure 4: Grid size in wall unit for the first layer grid on the cylinder.
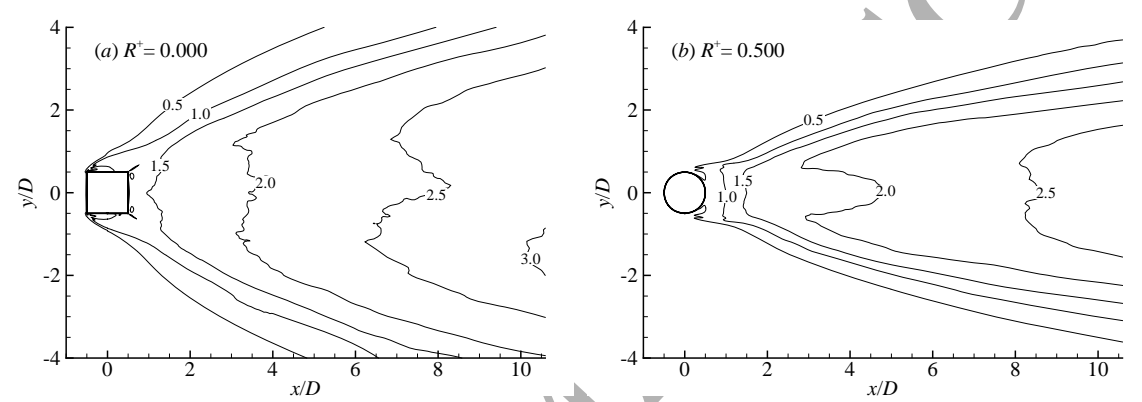

Figure 5: Ratio of the local grid size to the estimated Kolmogorov length scale $h / \eta_{k}$.

that of the circular cylinder hence omitted here for brevity. For the square cylinder, the maximum grid size in wall unit appears at the cylinder front corner $\left(45^{\circ}\right)$ where the flow separates from the surface. However, the unsteadiness of the flow is weak in this region, hence the relatively larger grid size does not deteriorate the simulation quality. On the lateral and rear surfaces of the cylinder the maximum $\Delta \eta^{+}$is smaller than unity, reflecting a well-resolved boundary layer flow in the turbulent regime. For the circular cylinder, since the attached flow naturally separates from the cylinder surface rather than the forced separation in its square counterpart, there are no sharp peaks in the profiles and the maximum $\Delta \eta^{+}$is always smaller than unity from the front to the rear stagnation point. 

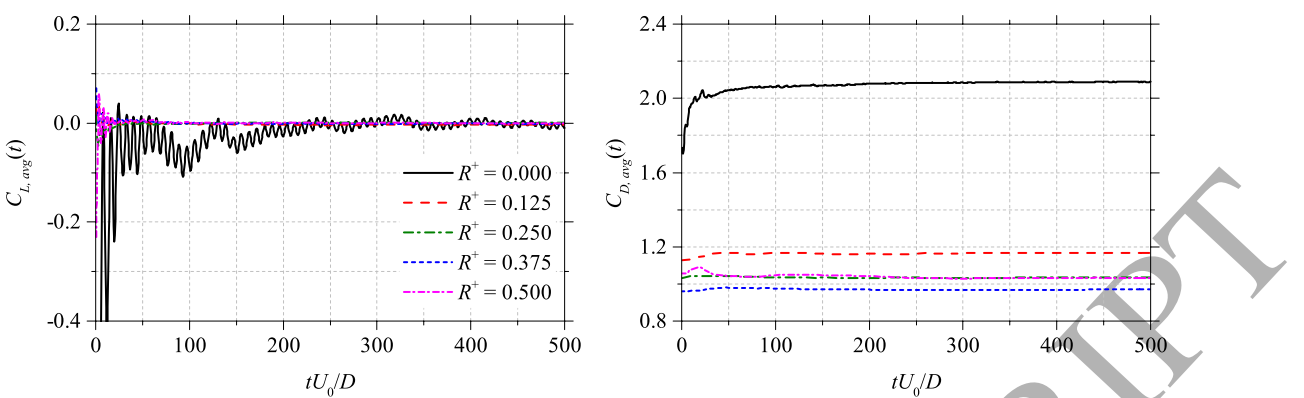

Figure 6: Time histories of the mean lift and drag coefficients as functions of integration time.
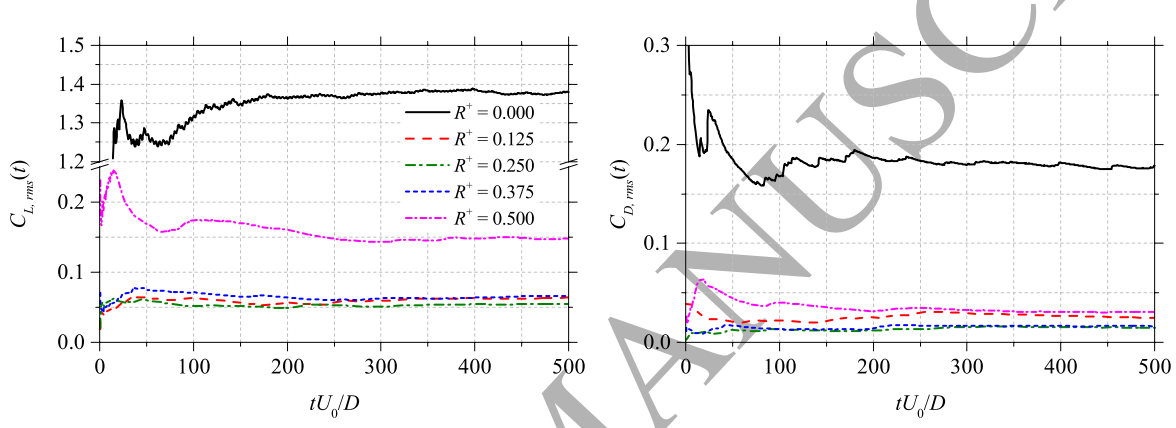

Figure 7: Time histories of the root-mean-square values of the fluctuating lift and drag coefficients as functions of integration time.

The spatial resolution required for resolving the turbulent flow is evaluated by the ratio between the grid size $(h)$ and the estimated Kolmogorov length scale $\left(\eta_{k}\right)$, as presented in Figure 5 for the square $\left(R^{+}=0.000\right)$ and circular $\left(R^{+}=0.500\right)$ cylinder cases, with the two quantities defined as:

$$
h=(\Delta \xi \Delta \eta \Delta z)^{1 / 3}, \eta_{k}=\left(v^{3} / \epsilon\right)^{1 / 4}, \epsilon=2 v \overline{S_{i j}^{\prime} S_{i j}^{\prime}},
$$

where $S_{i j}^{\prime}$ is the symmetric part of $\nabla \mathbf{u}^{\prime}$. Due to the clustering of the grid and the less developed turbulent flow, the ratio is close to or even smaller than unity in the region close to the cylinder. The grid size is larger and is about three times that of the 
Kolmogorov length scale in the region $x / D \geqslant 10.0$. Although this ratio is not a strong guarantee, nonetheless, the grid of this size is confirmed sufficiently fine to resolve the essential small-scale structures of the turbulent flow past a bluff body [58-60] and is considered fine enough in our study.

The sufficiency of the simulation duration is assessed by the time-averaged sectional lift and drag coefficients as a function of the integration time. The quantities are defined as:

$$
\begin{aligned}
& C_{L, a v g}(t)=\frac{1}{t-t_{0}} \int_{t_{0}}^{t} \frac{\mathrm{Lift}}{\frac{1}{2} \rho U_{0}^{2} D L_{z}} d t, \\
& C_{D, a v g}(t)=\frac{1}{t-t_{0}} \int_{t_{0}}^{t} \frac{\mathrm{Drag}}{\frac{1}{2} \rho U_{0}^{2} D L_{z}} d t,
\end{aligned}
$$

where $t_{0}<t \leq t_{1} ; t_{0}=0$ and $t_{1}=500 D / U_{0}$ are the start and end of the production simulation; $\rho=1$ is the constant density of the fluid. Both the pressure and viscous forces contribute to the lift and drag. The two quantities should converge to constant values as the integration time becomes sufficiently long [60]. The variations of the two quantities with respect to time are given in Figure 6. Both the lift and drag coefficients rapidly converge to constant values except for the lift at $R^{+}=0.000$ due to the large fluctuation amplitude (see Figure 9). The same assessment is also conducted for the root-mean-square values of the fluctuating lift and drag coefficients. The quantities are computed using Eqs. (10)-(11) by substituting the lift and drag with their fluctuating parts, and the results are shown in Figure 7. The R.M.S. fluctuations converge slower than the time-averaged values, but still achieve temporal convergence after the whole production simulation $\left(500 D / U_{0}\right)$. We believe the duration of $500 D / U_{0}$ is sufficiently

290 long for the data collection and statistics in present simulations. 

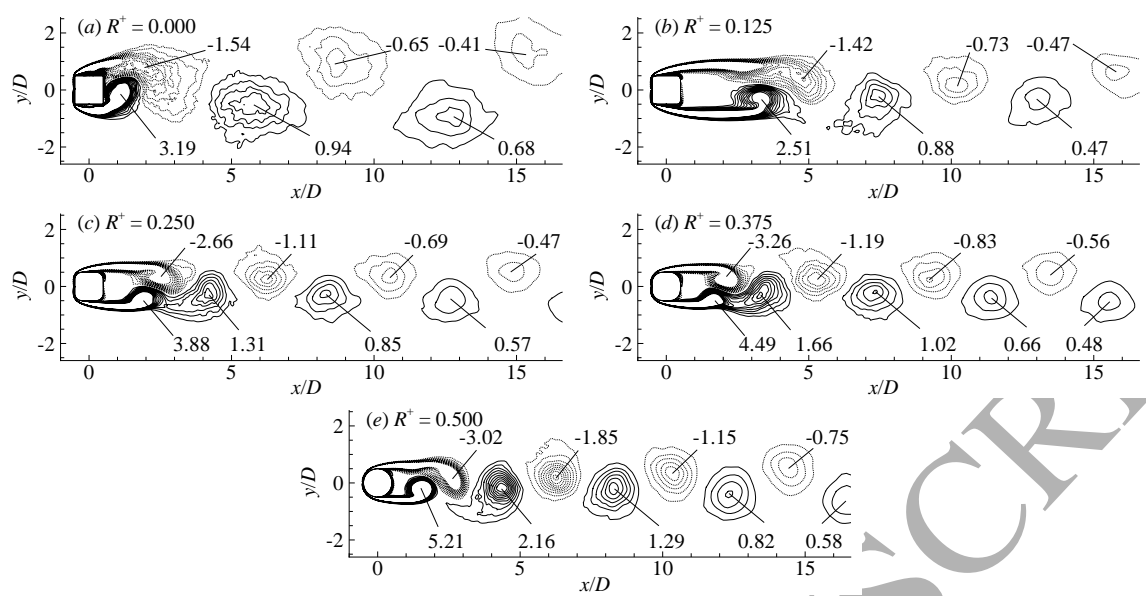

Figure 8: Instantaneous phase-averaged spanwise vorticity $\omega_{z} D / U_{0}$ plotted with an interval $\Delta \omega_{z} D / U_{0}=0.2$.

\section{Results and discussion}

\subsection{General description of the unsteady flow}

The separation of the flow and the time-periodic shedding of the wake vortices are depicted in Figure 8 using the instantaneous phase-averaged spanwise vorticity field. The flow pattern is largely affected by the corner radius of the cylinder. For the square cylinder $\left(R^{+}=0.000\right)$, the flow separates at the front corners of the cylinder, and the shedding of the wake vortices is observed downstream and close to the rear corners. The periodic shedding commences close to the cylinder at $x / D=1-2$. By slightly rounding the corners of the cylinder to $R^{+}=0.125$, the flow separation at the front

soo corners moves further leeward. The free shear layer is more stable and extends further downstream, and the roll-up of the wake vortices is initiated far from the cylinder at $x / D=3-5$. As $R^{+}$further increases, the free shear layer becomes less stable and the formation of wake vortices moves upstream eventually to be around $x / D=2-3$ 
$c_{t}$

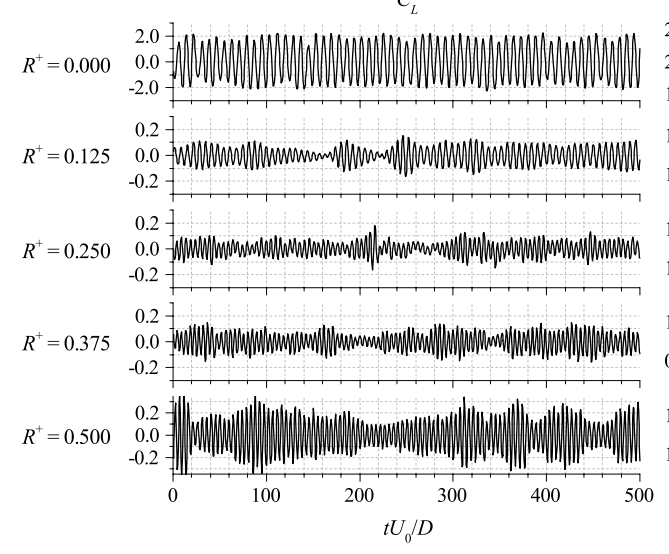

Figure 9: Time histories of the sectional lift and drag coefficients.

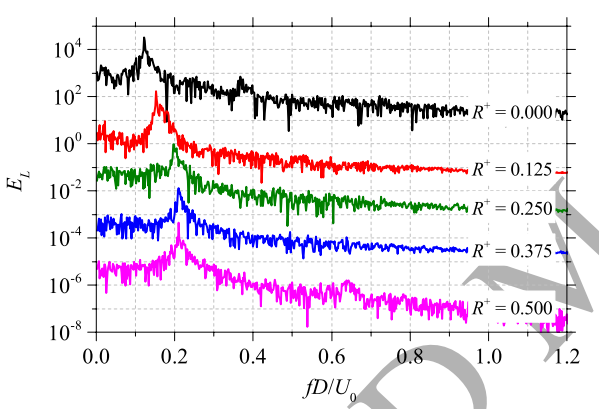

ag coefficients.
$C_{D}$

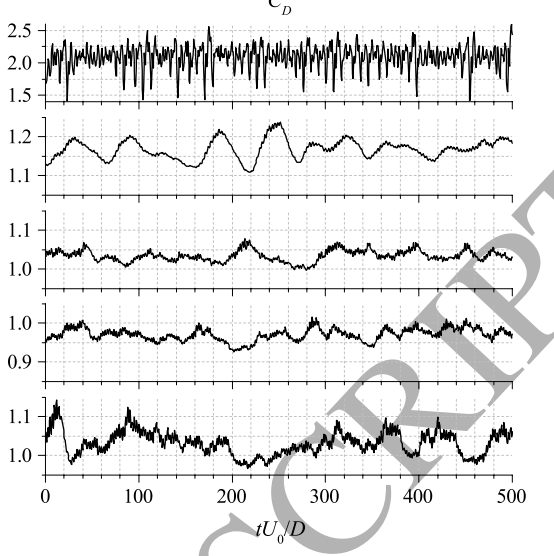

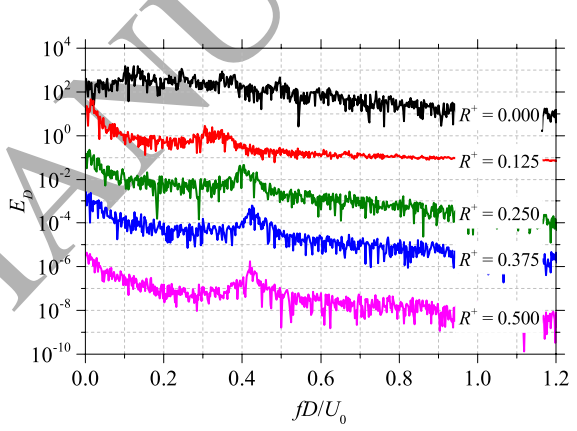

Figure 10: Temporal power spectra of the sectional lift and drag coefficients. The curves are artificially separated for clarity.

for the eircular cylinder $\left(R^{+}=0.500\right)$. The formation length of the shedding vortices,

roughly defined as the streamwise distance between them and the cylinder, varies inversely with the base pressure $-C_{p b}$ (refer Table 3), which is in accordance with the conclusion of Bearman [61]. The effect of $R^{+}$is clearly observed by comparing the vorticity fields. The first is the formation of the shedding wake vortices as described above; an abrupt delay of the formation position occurs by slightly rounding the cylinder corners to $R^{+}=0.125$, while the further increasing of $R^{+}$results in the formation 
of the vortices progressively at further upstream locations. The second difference is the distance between the wake vortices both in the streamwise and transverse directions; both distances get smaller as $R^{+}=0.000$ increases to $R^{+}=0.250$, and then remain almost unchanged as $R^{+}$further increases. The third difference is the intensity of the vortices, as measured by the vorticity magnitude at the center of the vortex. The unsteady flow is diffused by the fluid viscosity and the peak vorticity magnitude decays as the vortices convect downstream. Compared with that of the square cylinder, the vorticity of the first wake vortex is weaker for the rounded cylinder at $R^{+}=0.125$, but increases with $R^{+}$since the vortex is formed closer to the cylinder where the wake

flow is strongly recirculating. The flow pattern will be further analyzed in subsequent sections 3.2 and 3.3 .

The effect of $R^{+}$on the time-averaged characteristic quantities is tabulated in Table 3. Consistent with the observation in Figure 8, the Strouhal number monotonically increases with $R^{+}$but is nearly constant for $R^{+} \geqslant 0.250$. There is a sudden decrease of the drag and the force fluctuations as the cylinder is rounded at $R^{+}=0.125$, with a $50 \%$ decrease for the drag and even more for the force fluctuations. For the rounded cylinders, the force fluctuations are significantly smaller compared with the square cylinder case, since the flow is only notably unsteady in the far-wake but is relatively steady close to the cylinder due to the stable free shear layer. However, the force fluctuations ${ }_{330}$ eventually increase for the $R^{+}=0.500$ cylinder since the shedding of the vortices starts in the near-wake and the flow around the cylinder is more perturbed with large vorticity (see Figure 8(e)). For flow separation from the cylinder, the separation angle is exactly $45^{\circ}$ for the square cylinder due to the forced separation. As $R^{+}$increases, although the 
front corners are rounded and no forced separation is triggered, the flow separation for the partially rounded cylinders occurs at the front rounded corner (between $A_{0}$ and $A_{1}$ in Figure 2), while it is observed on the rear side for the circular cylinder at a separation angle slightly above $90^{\circ}$.

The time histories of the sectional lift and drag coefficients are given in Figure 9. For the square cylinder, the variation of $C_{L}$ is nearly perfect time-periodic, while $C_{D}$ shows more fluctuations and is noisier than those of other geometries. Referring to Figure 8 , it is seen that the wake vortex shedding largely determines the flow field at the rear side of the square cylinder. The vorticity isolines indicate that the vortices fluctuate probably due to their entrainment towards the wake centerline, which leads to the noisy $C_{D}$ variation. For the rounded corner cylinders, the wake vortices start to shed further away from the rear surface compared with the square cylinder, and hence the near-wake flow field is less affected. Consequently, the $C_{D}$ variations show smaller fluctuations in magnitude. Both force coefficients exhibit different patterns as $R^{+}$increases: the fluctuation amplitudes notably decrease and low-frequency modulations are observed, especially for the drag coefficient. This quasi-periodic modulation, namely, the beat phenomenon, is also observed for the low-Re flow past a circular or square cylinder (e.g., [19, 20, 37]) or a normal flat plate [42] attributed to the multifrequency components around the Karman vortex shedding frequency. It is observed in previous numerical studies that this beat phenomenon is only noticeable in threedimensional simulations as long as the spanwise domain size is sufficiently large [37] 355 and the spatial resolution is sufficiently high [19], in contrast to the (false) regular timeperiodic fluctuation observed in simulations with low aspect ratio or low resolution. It 
is important to note that this low-frequency modulation necessitates a longer duration of the simulation in order to get accurate prediction of force fluctuation, and this modulation may well be responsible for the variability of the predictions in the literature (see Table 3). Referring to Table 1, it is seen that the spatial resolution and the duration of our simulation are sufficiently large and we believe the results listed in Table 3 are reliable.

The temporal power spectra of the lift and drag coefficients are shown in Figure 10. Although the beat phenomenon is noticeable in the time history of the lift coefficient for all rounded corner cylinders, the lift spectrum exhibits a single peak corresponding to the periodic vortex shedding frequency for all cylinders. The single peak in the drag spectrum is inconspicuous and could only be clearly identified for $R^{+} \geqslant 0.250$, where the corresponding frequency is twice as that of the lift. The amplitude of the peak is roughly equal, if not smaller than the low frequency part. This finding is different from that observed by Sohankar et al. [20] for flow past a square cylinder at $R e=500$ in which the drag coefficient shows a quasi-periodic pattern and a single peak is observed in its power spectrum. The low frequency modulation gets pronounced for $R e>500$ and is responsible for the missing peak in the drag spectra in our study.

3.2. Time-averaged results

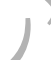

We first examine the effect of the corner radius on the time-averaged flow. Figure 11 shows the time-averaged normalized spanwise vorticity field. The flow separates at the front corners of the square cylinder $\left(R^{+}=0.000\right)$ and tiny recirculation bubbles form on the lateral surfaces; the vorticity field indicates that the time-averaged recirculation bubble in the wake is small in the streamwise direction. At $R^{+}=0.125$, the free shear 

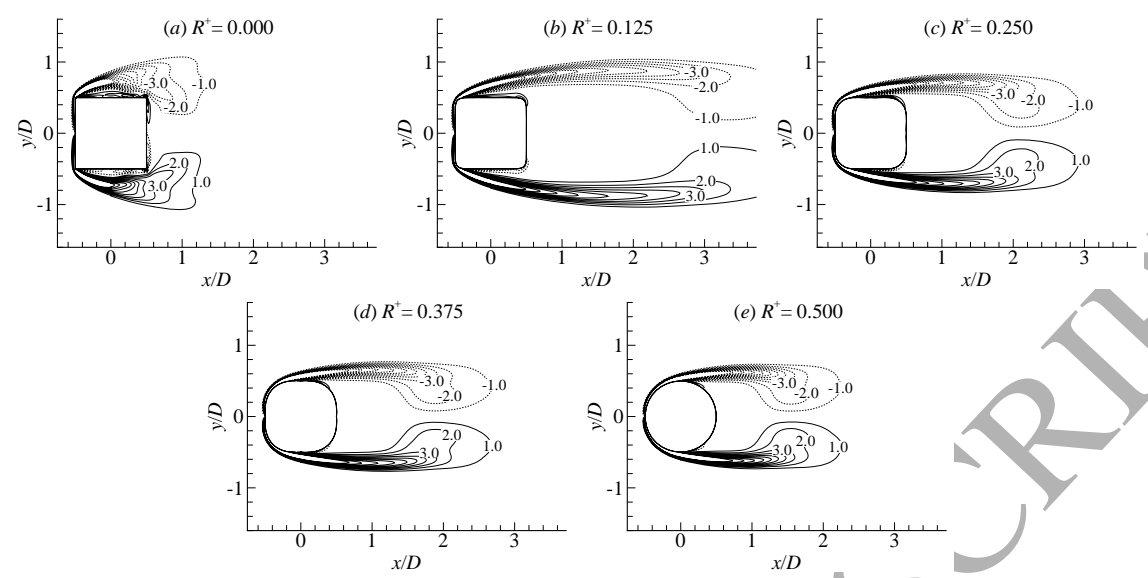

Figure 11: Time-averaged spanwise vorticity $\omega_{z} D / U_{0}$ plotted at $\Delta \omega_{z} D / U_{0}=1$.

layer extends far away from the cylinder and shøws signs of roll up into wake vortices at around $x / D=3.0$. The angle between the separated free shear layer and the lateral surface decreases at $R^{+}=0.250$, and the wake recirculation bubble gets smaller in both streamwise and transverse directions. As $R^{+}$further increases beyond 0.250 , the separation angle of the free shear layer remains almost unchanged, and the recirculation bubble becomes noticeably smaller only in the streamwise direction but not so obvious in the transverse direction.

The time-averaged wake flow is further depicted by the streamwise velocity as shown in Figure 12. The profiles illustrate the variation of the time-averaged wake flow in both directions. Since experimental data at $R e=1,000$ is scarce, the profiles are compared with those of flow past a circular cylinder at $R e=3,900$ measured by Lourenco \& Shih and Ong \& Wallace [63]. It is seen that the effect of the Reynolds number is pronounced in the near-wake that the profiles do not match well, while very good agreement is achieved starting at $x / D=3$ up until the far-wake. The velocity 


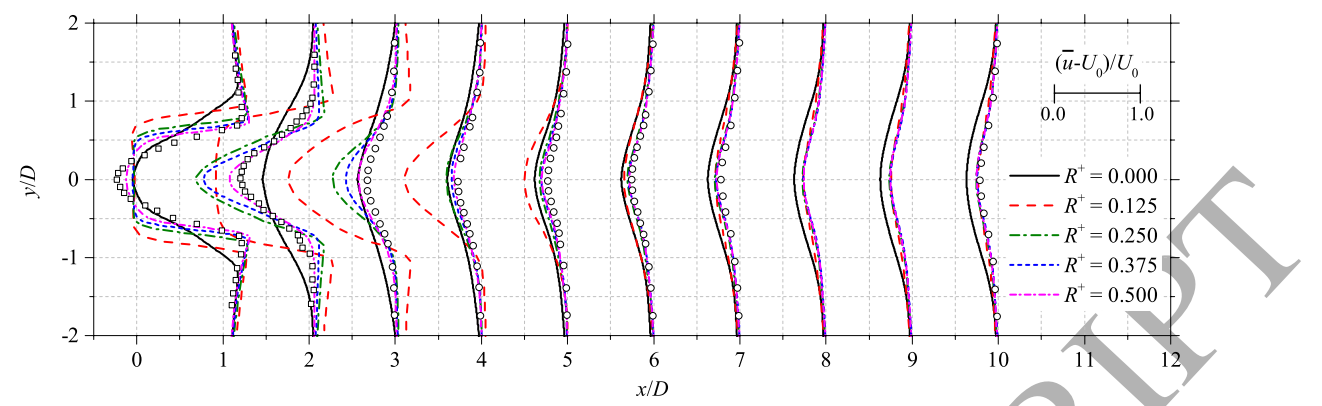

Figure 12: Profiles of the time-averaged streamwise velocity $\left(\bar{u}-U_{0}\right) / U_{0}$. The hollow squares and circles are the experimental results for circular cylinder $\left(R^{+}=0.500\right)$ case at $R e=3,900$ by Lourenco \& Shih (extracted from Kravchenko \& Moin [62]) and Ong \& Wallace [63], respectively.

deficit in the wake is denoted by the negative value in the profile, while reversed flow

in the recirculation bubble is represented by $\left(\bar{u}-U_{0}\right) / U_{0}<-1$. The deficit is significant within the recirculation bubble, exhibiting a typical parabolic profile whose transverse extension depends on the cylinder geometry. The near-wake flow for the $R^{+}=0.125$ cylinder presents a flat profile since it is dominated by the recirculating flow, and its recovery in the streamwise direction is the slowest as a result of the large wake recirculation bubble. The width of the wake, roughly represented by the deficit streamwise velocity in the transverse direction, gets larger along the streamwise direction as a result of the viscous diffusion. It is noted that the velocity deficit in the far-wake of the square cylinder is much more pronounced than the others and the wake is wider.

The distributions of several time-averaged characteristic quantities are presented in Figure Figure 13, and comparative validations with available experimental $[27,56,62]$ and numerical $[36,37]$ data are provided for cases with Reynolds number that is closest to our simulation. It is seen from the pressure coefficient distribution that there is a favorable pressure gradient (FPG) field along the circumference from the front stagnation point 

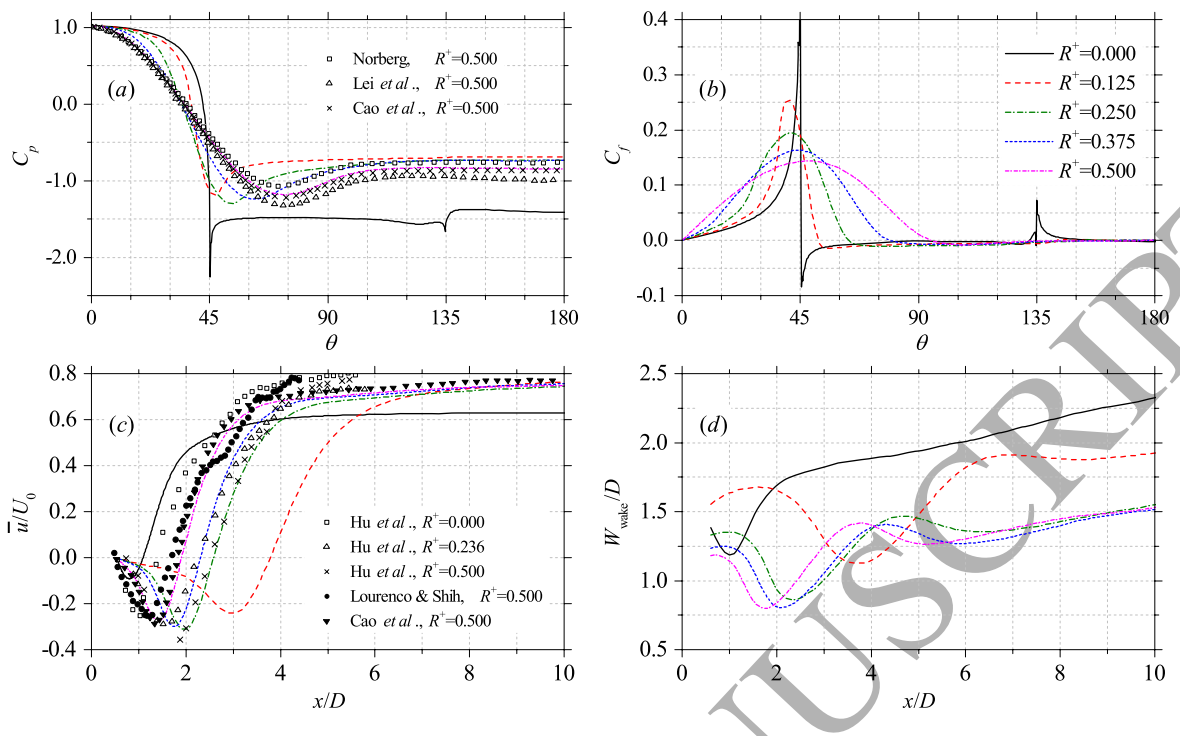

Figure 13: Spatial variations of several time-averaged quantities: (a) pressure coefficient along the circumference of the cylinder; $(b)$ skin friction coefficient along the circumference of the cylinder; $(c)$ streamwise velocity along the horizontal wake centerline $(y / D=0)$ downstream of the cylinder; $(d)$ width of the wake along the horizontal wake centerline downstream of the cylinder. The present results are compared with available data by Norberg [56] (experimental, $R e=1,500$ ), Lei et al. [37] (numerical, $R e=1,000$ ), Cao et al. [36] (numerical, $R e=1,000)$, Hu et al. [27] (experimental, $R e=2,600$ ) and Lourenco \& Shih (experimental, $R e=3,900$, extracted from Kravchenko \& Moin [62]). $\theta$ is the azimuthal angle measured from the front stagnation point of the cylinder, as shown in the rightmost subfigure in Figure 2.

to a location at or before the separation point, followed by an adverse pressure gradient (APG) field and then a pressure plateau after separation. The intensity of the FPG and APG fields, indicated by the magnitude of the local pressure gradient, decreases with $R^{+}$. For the circular cylinder, the pressure coefficient reaches that of the cross-flow $\left(p_{\infty}=0.0\right)$ at $\theta=35.5^{\circ}$ and the suction peak appears at $\theta=73.5^{\circ}$, which are in agreement with the experimental values of $36^{\circ}$ and $71^{\circ}$ for the $R e=1,500$ flow by Norberg 415 [56]. Since the fluid is accelerated by the FPG field and decelerated by the APG field, 
the corresponding skin friction coefficient varies accordingly as shown in Figure 13(b). The azimuthal variation of $C_{f}$ is milder as $R^{+}$increases. Although the rounding of the corners relieves the forced separation at the front corners, the flow still separates at the front rounded corner for the partially rounded cylinder $\left(R^{+}=0.125-0.375\right)$, as indicated by negative values of $C_{f}$.

The distribution of the normalized streamwise velocity $\bar{u} / U_{0}$ confirms the previous conclusion that the length of the wake recirculation bubble drastically increases by first changing $R^{+}=0.000$ to $R^{+}=0.125$, but then decreases with further increase of $R^{+}$. It is worth noting that $R^{+}$also affects the rate of recovery of the streamwise velocity to

the free stream value in the far-wake. The velocity in the wake of the square cylinder recovers slowly and is about $\bar{u} / U_{0}=0.63$ at $x / D=10.0$, while the velocity magnitude is larger and almost reaches the same value of 0,76 for all the rounded cylinders. This can be partially explained by the widening of the wake for the square cylinder, as shown in Figure $13(d)$. Here we define the wake width as the distance between the two critical points where the streamwise velocity deficit is $50 \%$ that of the maximum value [27], i.e., $\left(\bar{u}-U_{0}\right) / U_{0}$ at the wake centerline. Based on this definition, the wake width is entirely determined by the local velocity profile. For the near-wake flow, the velocity profile changes from flat to parabolic shape along the streamwise direction, thus the critical points move towards the horizontal wake centerline and the wake width reduces. As the flow develops downstream, the streamwise velocity becomes more uniform in the $y$-direction; the critical points move further away from the horizontal wake centerline and increases the wake width. The wake width of the square cylinder is the largest among the several geometries since the maximum streamwise velocity 
deficit is the largest (see Figure 12).

Our simulation results are generally consistent with those in the literature. The azimuthal distribution of $C_{p}$ is verified by the DNS result of Lei et al. [37] and LES result of Cao et al. [36] for the circular cylinder case at $R e=1,000$, while is slightly different from the experimental result of Norberg [56] which is attributed to slightly different Reynolds number in the experiment. The spatial resolution has an obvious effect on the $C_{p}$ distribution; our result obtained using the cross-sectional $512 \times 512$ grid agrees very well with that of Cao et al. [36] using the $160 \times 140$ grid, while there is a $10 \%-20 \%$ difference for the result of Lei et al. [37] due to their smaller $81 \times 81$ grid. The streamwise distribution of $\bar{u} / U_{0}$ in the wake is also verified, especially for the circular cylinder case as compared with the result of Cao et al. [36]. However, it is noted that in the low-Re regime, the slight variation of the Reynolds number leads to completely different wake flow that the velocity distribution is greatly affected.

Based on the above analyses, it is generally concluded here that the corner radius has great effect on the time-averaged flow. The flow past the square cylinder experiences forced separation at the sharp corners and a relatively smaller recirculation bub-

455 ble forms in the wake. There is a significant change of the time-averaged flow as the corners of the square cylinder are rounded at $R^{+}=0.125$; the free shear layer is more stable and extends further away from the cylinder before the onset of vortex shedding, thus results in a large recirculation bubble. As $R^{+}$further increases, the angle between the separated free shear layer and the cylinder lateral surface becomes smaller and so 460 does the wake recirculation bubble in both streamwise and transverse directions. The magnitude of the wake flow velocity recovers to that of the incoming flow in the far- 

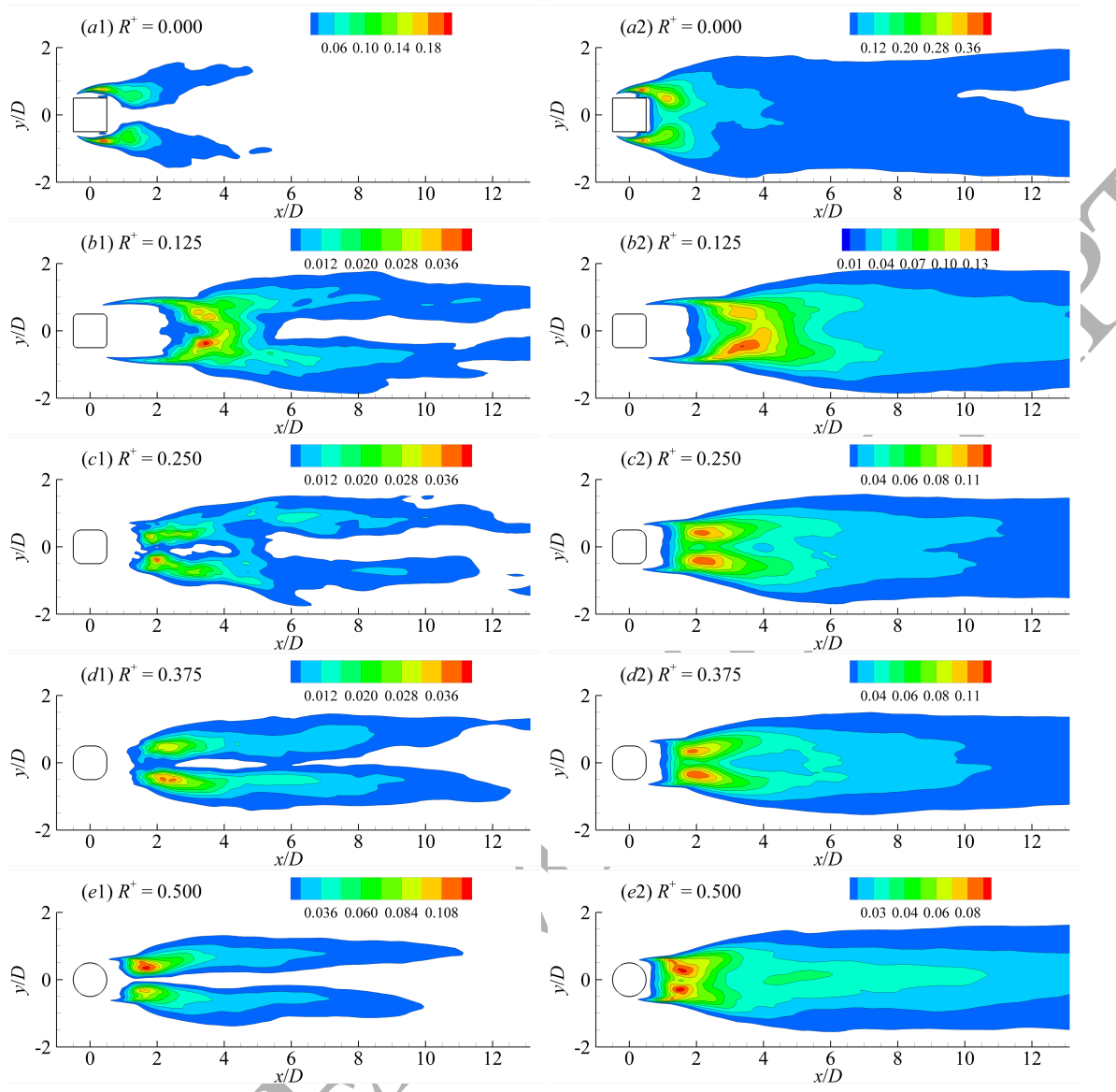

Figure 14: Time-averaged normal Reynolds stress contributed by: (a1-e1) coherent velocity $\overline{u^{*} u^{*}} / U_{0}^{2} ;(a 2$ $e 2)$ incoherent velocity $\overline{u^{\prime} u^{\prime}} / U_{0}^{2}$.

wake for the rounded cylinders, while the recovery is slowest for the square cylinder which has the widest wake width.

\subsection{Time-dependent results}

The incoming steady laminar flow past the cylinder loses its stability at the present Reynolds number $R e=1,000$, and is characterized by the time-dependent behavior 

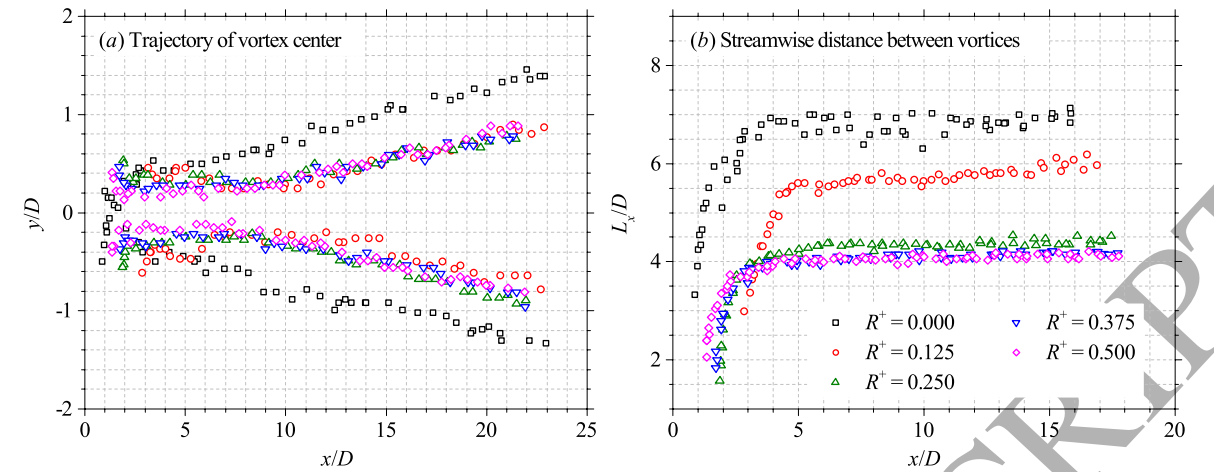

Figure 15: Vortex center measured from the phase-averaged flow field: (a) trajectory of the centers of the multiple wake vortices (see Figure 8); (b) streamwise distance to the downstream neighbor vortex.
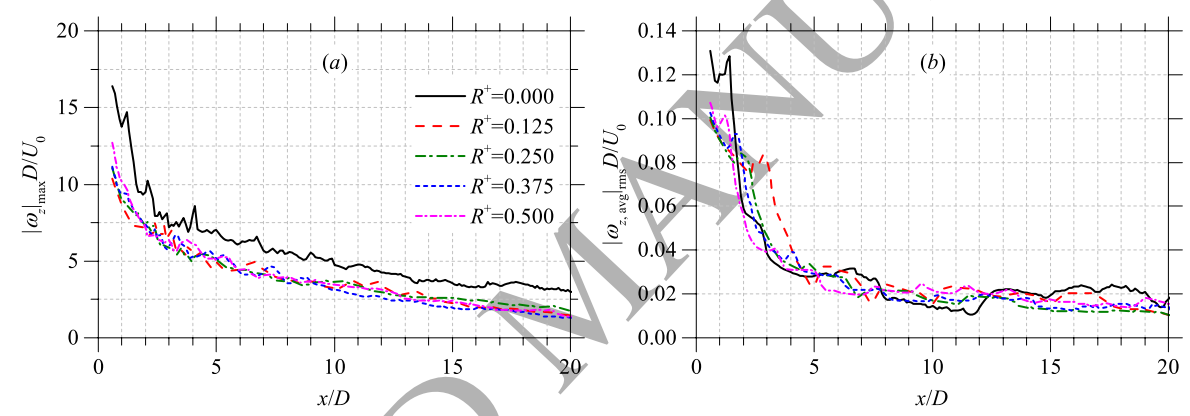

Figure 16: Downstream decay of the maximum and the cross-section averaged spanwise vorticity.

and fluctuating forces acting on the cylinder, and the unsteadiness in the wake. For a general depiction of the unsteady flow, Figure 14 shows the spatial distributions of the contributions of the coherent and incoherent streamwise velocity on the Reynolds stress (i.e., $\overline{u^{*} u^{*}} / U_{0}^{2}$ and $\overline{u^{\prime} u^{\prime}} / U_{0}^{2}$ ), computed through Eqs.(5)-(8). The coherent stress component represents the time-periodic behavior of the flow as a result of the primary wake instability, while the incoherent one reflects the turbulent fluctuation and the lowfrequency modulation of the flow (see Figure 9). The Reynolds stresses are pronounced in the free shear layer and near-wake but gradually decay downstream. For the square 
Table 4: Convection velocity of the vortices in the far-wake region.

\begin{tabular}{cccc}
\hline Source & Re & Geometry & Convection velocity $/ U_{0}$ \\
\hline Cantwell \& Coles [44] & 140,000 & Circular & 0.85 \\
Tanaka \& Murata [64] & 37,000 & Circular & $0.81-0.83$ \\
Zhou \& Antonia [65] & 5,600 & Circular & $0.86-0.92$ \\
Present & 1,000 & $R^{+}=0.000$ & $0.79-0.85$ \\
& & $R^{+}=0.125$ & $0.85-0.91$ \\
& & $R^{+}=0.250$ & $0.85-0.89$ \\
& & & $0.82-0.86$ \\
& & $R^{+}=0.375$ & $0.82-0.86$
\end{tabular}

cylinder, the fluctuation grows in the free shear layer from the front corners, and is the most significant at the rear corners due to vortex shedding and the entrainment towards the wake; both the coherent and incoherent stresses are also pronounced in the nearwake region $1 \leqslant x / D \leqslant 2$ as a result of first vortex shedding and the fluctuations in the recirculating flow. The shear layer is more stable as the cylinder is rounded at 480 $R^{+}=0.125$ and the stresses start to grow close to the rear corners; both stresses are the most significant at the reattachment point (downstream limit of the recirculation bubble [47]) where the recirculating flow vanishes. As $R^{+}$further increases, the growth of the stresses is weak in the shear layer but only pronounced close to the reattachment pojnt (see Figure 11). It is evident that apart from the spatial distribution, the Reynolds stresses are also affected by $R^{+}$in terms of their intensities. For the square cylinder, the maximum magnitude of the coherent Reynolds stress is noticeably smaller than that of the incoherent component due to the larger stochastic fluctuation and low-frequency 

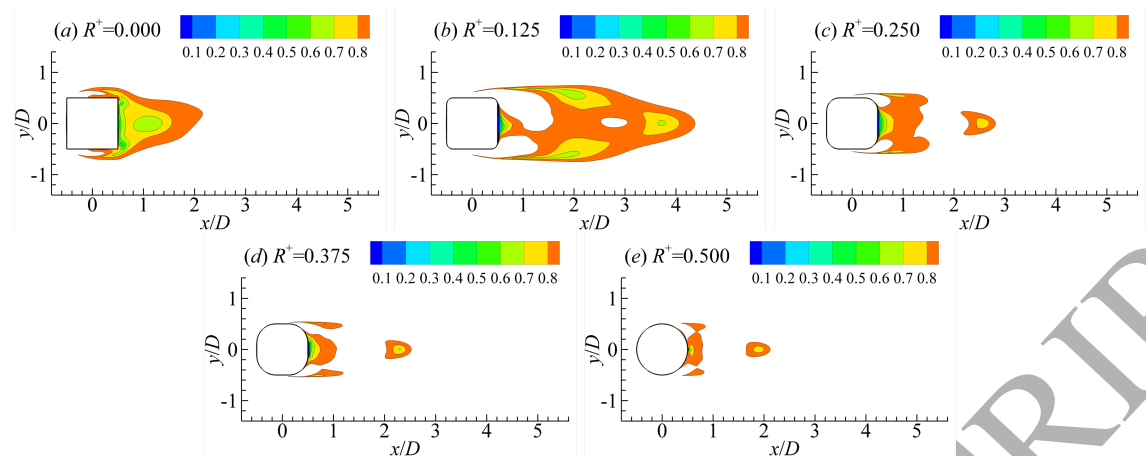

Figure 17: Two-time correlation coefficient of the streamwise velocity

modulation; this is observed in a small region close to the rear corners where the flow can not be viewed as perfectly time-periodic even in the present low-Re regime. Both

the coherent and incoherent Reynolds stresses are notably reduced close to the cylinder surfaces, especially on the lateral walls where the pressure difference dominates the lift. Since the flow fluctuation is weaker as $R^{+}$increases, both the coherent and incoherent Reynolds stresses decrease but the incoherent component is still dominant, reflecting an imperfect time-periodic flow. The intensities of the stresses do not change much for the rounded cylinders, but exhibit a great variation for the circular cylinder: there is a drastic increase of the maximum magnitude of the coherent stress and a decrease of the incoherent one, and the former is larger in the wake where the downstream motion of the shed vortices is significant. The near-wake flow of the circular cylinder case is the most regular in time compared with other geometries.

The time-dependent motion of the shedding vortices is analyzed in Figure 15. The trajectory of the phase-averaged vortex center is identified from a number of snapshots in about one shedding cycle. The vortices shed from the shear layer first experiences 
an inwards entrainment towards the horizontal wake centerline $(y / D=0.0)$, which is somewhat obvious as the first roll up of the wake vortex. As the flow develops downstream, the wake vortices in the square cylinder case translate outwards away from the horizontal centerline and a wider wake forms; however, the vortices of the rounded cylinder cases first translate mostly in the streamwise direction without notable transverse motion in the region upstream of $x / D=10.0$, then move outwards which expands the width of the wake. The vortices of the flow past the square cylinder are relatively far away from the horizontal wake centerline, while the trajectories for the rounded cylinder cases are almost the same and closer to the horizontal wake centerline. In addition to the transverse motion of the wake vortices, Figure $15(b)$ shows the streamwise distance between them. The distance is small in the near-wake due to the deceleration by the recirculating flow (refer Figures 8 and 13), while it reaches a constant value roughly downstream of $x / D=5.0$ for all cylinders, i.e., outside of the wake recirculation bubble. The corner radius has a less significant effect on the vortex separation distance as $R^{+}$increases. It is noted that the streamwise distance between neighboring vortices depends on $R^{+}$and reveals the convection velocity of the vortices in the far-wake region. The convection velocity may be estimated by:

$$
f L_{x}=\frac{S t U_{0}}{D} L_{x}
$$

Referring to the Strouhal number in Table 3, the convection velocity of the vortices are computed and listed in Table 4 . We find that $R^{+}$has a small effect on the convection velocity: the velocity is the smallest for the square cylinder, but increases for the $R^{+}=$ 0.125 cylinder probably due to the weak recirculating flow where the wake vortices are less decelerated (see Figure 13(c)); the velocity only slightly decreases as $R^{+}$further 
increases. The present results, in comparison with those in the literature listed in Table 4, are also consistent with the conclusion of Zhou \& Antonia [65] that the convection velocity is almost independent of the Reynolds number.

As shown earlier in Figure 8, the magnitude of the spanwise vorticity measured at the center of the shedding vortices decays as convecting downstream due to the viscous diffusion. The magnitude of the phase-averaged spanwise vorticity, in terms of its maximum value and cross-section averaged value, as a function of streamwise distance are shown in Figure 16. Since the flow far away from the cylinder is less perturbed and can be considered steady and uniform, the cross-section averaged value is computed within the range $-5.0 \leqslant y / D \leqslant 5.0$. The maximum vorticity for the rounded cylinders is almost not affected by $R^{+}$, while it is obviously smaller than that of the square cylinder in the whole wake due to the slow recovery and consequently large strain. The effect of $R^{+}$on the cross-section averaged spanwise vorticity is not as notable as the maximum value. A larger averaged vorticity is observed for the square cylinder case only around the region $x / D \leqslant 1.5$; in addition to the peak value around $x / D=0.5$, there is a second peak at $x / D=1.3$ due to the formation and entrainment of the first shedding vortex. The same variation pattern is also observed for the rounded cylinders except for the position of the second peak depending on the initial shedding of the vortices.

The time-periodicity and temporal dynamics of the unsteady flow are further investigated by the two-time correlation of the streamwise velocity defined as:

$$
C_{u}(x, y)=\frac{\overline{u(x, y, t) \cdot u(x, y, t+T)}}{\sqrt{\overline{u(x, y, t)^{2}}} \sqrt{\overline{u(x, y, t+T)^{2}}}},
$$

in which $u$ is the instantaneous spanwise-averaged streamwise velocity; $T$ is the nor- 
malized period of the pulsating flow field computed from the Strouhal number in Table

3. The coefficient is unity in regions where the flow is steady or perfectly $T$-periodic, while it is less than one where the flow is not time-periodic due to turbulent fluctuations, phase jitter and low-frequency modulation, and could even be negative if the flow is temporally correlated in an anti-phase mode. The coefficient $C_{u}$ is computed from $t=0$ to $t=500 \mathrm{D} / U_{0}$ for all geometries for a statistically time-invariant representation of the flow field, which is about 60 cycles for the square cylinder case and 100 cycles for the circular cylinder case, depending on the period shown in Table 3. The distributions of the coefficient $C_{u}$ are plotted in Figure 17. The flow starts to be stochastic in the free shear layer since it is unstable to three-dimensional perturbations at the present Reynolds number. The temporal decorrelation is pronounced in the wake especially within the recirculation bubble and at the time-averaged reattachment point (see Figure 11). The decorrelated flow dominates the near-wake for the $R^{+}=0.125$ cylinder and is the most significant among all geometries. As $R^{+}$increases, the wake flow becomes more time-periodic and the area of the decorrelation is smaller; the flow past the circular cylinder is the most well organized with decorrelation appearing only at the rear stagnation point and the reattachment point.

The simulation results show that the flow starts to be unstable in the separated free shear layer and the fluctuation is the most pronounced in the near-wake, especially at the time-averaged reattachment point where the primary vortex shedding starts. The flow past the circular cylinder is the most regularized in the sense that time-periodicity is achieved for the majority region in the wake, and the coherent Reynolds stress $\overline{u^{*} u^{*}} / U_{0}^{2}$ is larger than the incoherent component $\overline{u^{\prime} u^{\prime}} / U_{0}^{2}$, representing a well orga- 

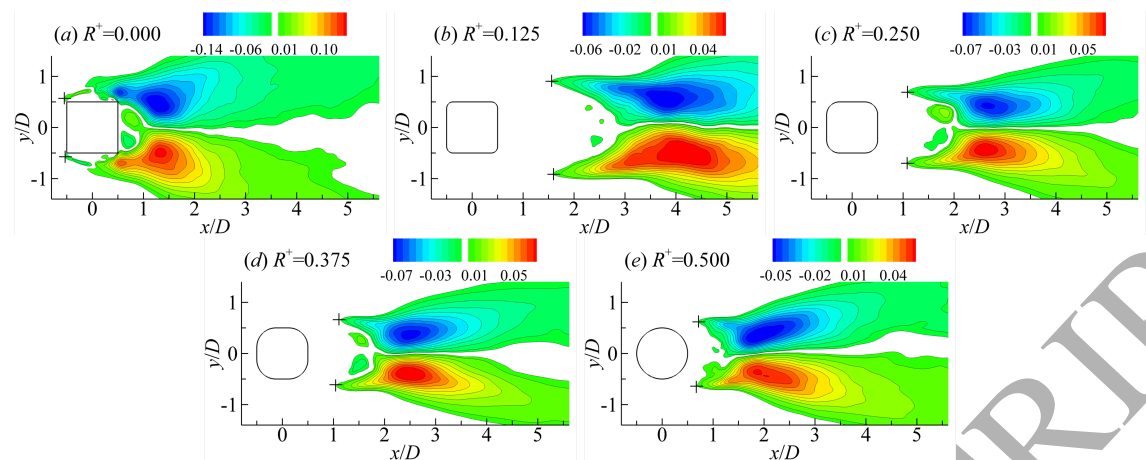

Figure 18: Spatial distribution of the shear Reynolds stress $\overline{u^{\prime} v^{\prime}} / U_{0}^{2}$. The plus symbol indicates the location of the transition onset at the threshold value $\overline{u^{\prime} v^{\prime}} / U_{0}^{2}= \pm 0.001$.

nized time-periodic flow. The shed vortices move further away from the horizontal wake centerline as they convect downstream, and the trajectory of the vortex center of the square cylinder case is distinctly different from other geometries. The corner radius $R^{+}$has negligible effect on the convection velocity of the shedding vortex in the far-wake, and the results are consistent with previous studies.

\subsection{Laminar-turbulent transition}

It is well known that the laminar-turbulent transition occurs at the separated free shear layer via the Kelvin-Helmholtz mechanism for flow past an isolated cylinder. From a numerical perspective, the transition onset can not be simply identified by the first appearance of non-zero $\mathbf{u}^{\prime}$ or $p^{\prime}$ since perturbations due to round-off error and numerical residual are always non-zero. A commonly used indicator for the transition onset is the time-averaged incoherent Reynolds stress component $\overline{u^{\prime} v^{\prime}} / U_{0}^{2}$. A threshold 560 value of the absolute magnitude of 0.001 is chosen to locate the transition onset in a certain number of numerical (e.g., [66, 67]) and experimental (e.g., [68, 69]) investiga- 
Table 5: Time-averaged enstrophy components computed within $500 \mathrm{D} / U_{0}$.

\begin{tabular}{ccccc}
$R^{+}$ & $E^{2} s_{x y}$ & $E n s_{z}$ & $E_{\text {tot }}$ & Ens $_{z} /$ Ens $_{\text {tot }}$ \\
\hline 0.000 & 0.0523 & 0.0386 & 0.0909 & 0.4246 \\
0.125 & 0.0310 & 0.0262 & 0.0572 & 0.4580 \\
0.250 & 0.0279 & 0.0250 & 0.0529 & 0.4726 \\
0.375 & 0.0268 & 0.0238 & 0.0506 & 0.4704 \\
0.500 & 0.0287 & 0.0223 & 0.0510 & 0.4373 \\
\hline
\end{tabular}

tions on flow past bluff bodies. Due to the symmetry of the cylinder geometry, here we use the criterion $\overline{u^{\prime} v^{\prime}} / U_{0}^{2}= \pm 0.001$ and plot the contour in Figure 18, where the transition onset is marked by the plus symbol. Two transition onset locations are observed

in the separated shear layer above the lateral sides for all cylinders, and are generally symmetric about the centerline $y / D=0$. For the square cylinder, the transition occurs close to the front corners where the flow is forced to separate from the cylinder. The onset is substantially postponed downstream to around $x / D=1.6$ for the $R^{+}=0.125$ cylinder, and are further away from the $y / D=0$ centerline in the transverse direction.

570 As $R^{+}$increases, the onset location moves upstream and gets closer to the cylinder in the transverse direction. Referring to Figure 8, we note that the transition is closely related with the location of the first shed vortex; the larger formation length of the wake vortices leads to later onset to transition. A more detailed discussion follows in the next section. 


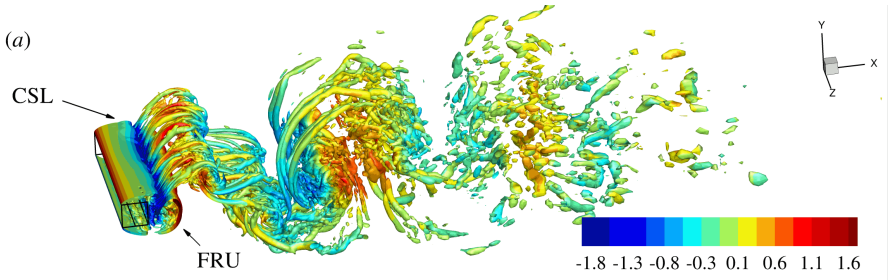

(b)

CSL

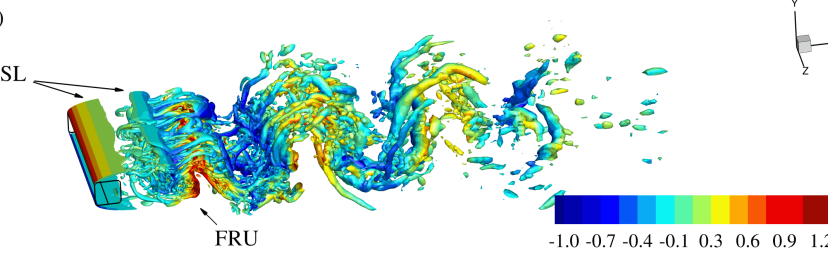

$\sum_{z}^{y}-$

(c)
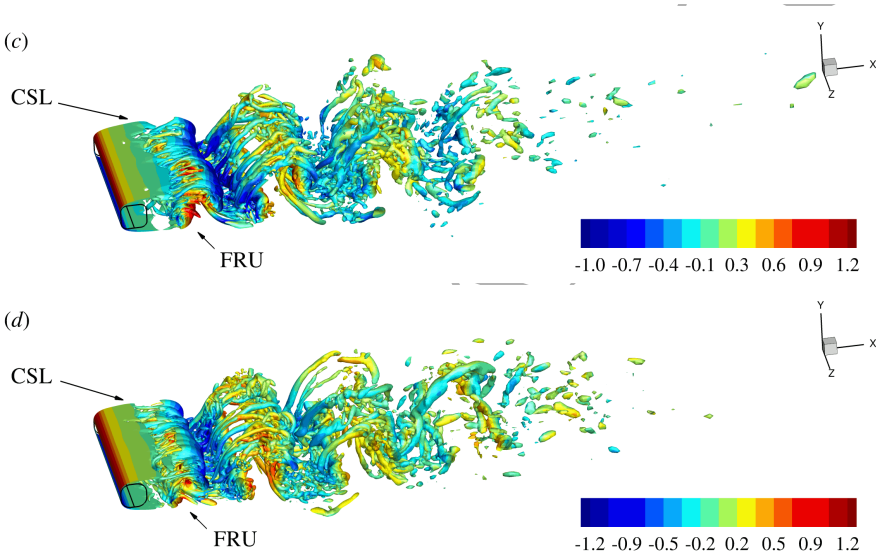

(e)

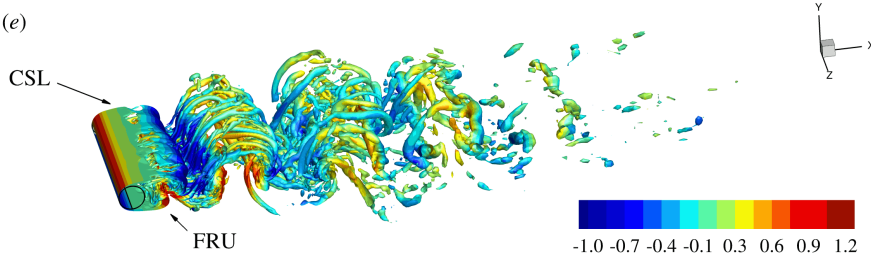

Figure 19: Instantaneous vortical structures represented by the isosurface of $Q=1$ and colored by the transverse velocity $v / U_{0}$ : (from $(a)$ to $\left.(e)\right) R^{+}=0.000,0.125,0.250,0.375$ and 0.500 . The corrugated shear

layer (CSL) and first roll up (FRU) of the wake vortices are denoted. 

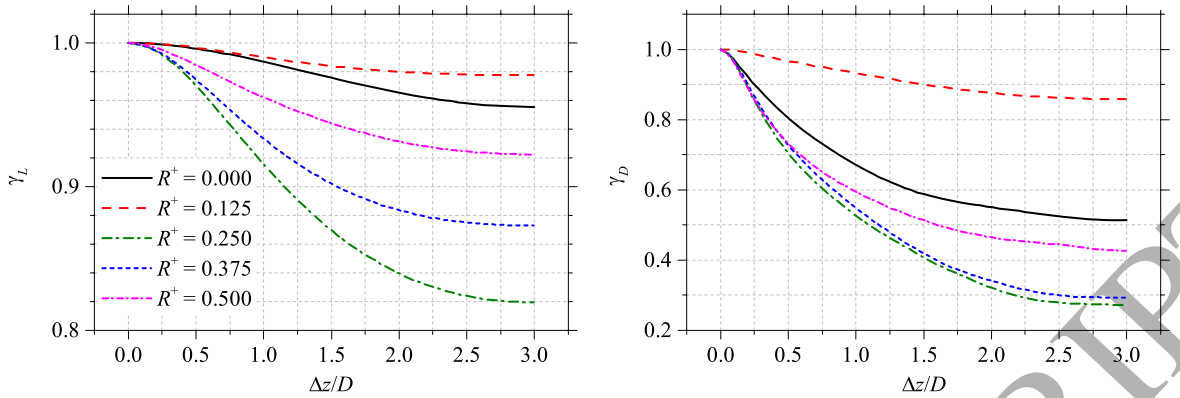

Figure 20: Spanwise two-point correlation of the fluctuating sectional lift and drag coefficients as a function of separation distance.

\subsection{Three-dimensional flow}

Although there is no spanwise skewness in the cylinder geometry nor spanwise force acting on the flow, the flow develops three-dimensionality at the present Reynolds number and spanwise non-uniformity emerges. This is demonstrated in Figure 19 showing the instantaneous three-dimensional flow structures, as represented by the $Q$ invariant [70]. The early stage separated flow in the form of a vortex (shear) layer is almost uniform along the spanwise direction. Since it is absolutely unstable to threedimensional perturbation [71], spanwise corrugated shear layer (CSL) appears, amplifying the large-scale 3D structures roughly at the streamwise position of the first roll up (FRU) of the wake vortex, and even smaller-scale structures further downstream. The breakdown from the large- to small-scale structures is accompanied by the vortex shedding in the near-wake, while the $3 \mathrm{D}$ wake flow is less regularized far away from the cylinder with periodic shedding not evident in the far-wake. The effect of the corner radius is obviously seen from the streamwise position of the appearances of the CSL and FRU. The separated shear layer over the square cylinder is the least resistant 

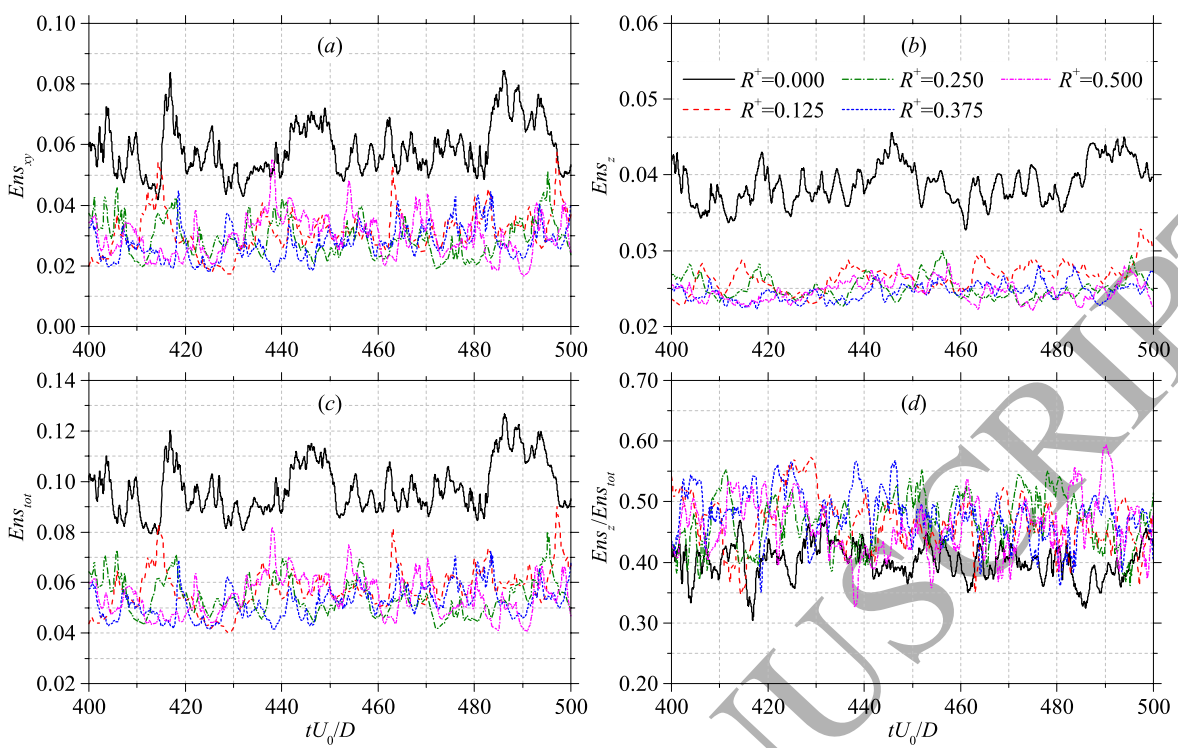

Figure 21: Temporal variations of the enstrophy components: $(a)$ secondary enstrophy $E n s_{x y} ;(b)$ primary enstrophy $E n s_{z} ;(c)$ total enstrophy $E n s_{t o t} ;(d)$ fraction of primary to total enstrophy $E n s_{z} / E n s_{t o t}$.

to the perturbation, and CSL appears even upstream of the rear corners. The wake flow entrains towards the rear side as it past the rear corners, resulting the immediate formation of FRU. The formations of CSL and FRU are postponed as the corner are rounded at $R^{+}=0.125$; the shear layer is more stable and remains spanwise uniform until relatively far away from the cylinder. As $R^{+}$further increases, both CSL and FRU move closer to the cylinder. Another observation made from comparing the subfigures is that the flow is the most strongly perturbed by the square cylinder in that the vortical structures are remarkable for a large extent in the transverse direction.

The effect of $R^{+}$on the three-dimensionality of the flow is quantitatively shown in Figure 20 with the spanwise two-point correlation of the fluctuating lift and drag 
coefficient. The coefficient is defined as:

$$
\gamma_{\phi}(\Delta z)=\frac{\overline{\phi^{f}(z, t) \phi^{f}(z+\Delta z, t)}}{\phi_{r m s}^{f}(z) \phi_{r m s}^{f}(z+\Delta z)},
$$

in which $\phi^{f}$ denotes the fluctuating sectional lift or drag coefficient (see Eq.(6)); $\Delta z$ is the spanwise separation distance between two sections. The coefficient is averaged over combinations of section pairs with the same $\Delta z$. The coefficient is unity if the fluctuating forces at the two sections are temporally varying in exactly the same pattern, while a smaller coefficient indicates spanwise non-uniformity as a result of the asynchronous variation, either in magnitude or phase. Compared with the square cylinder, the fluctuating forces acting on the $R^{+}=0.125$ cylinder show a stronger correlation in

the spanwise direction. This is attributed to the large formation length of the shedding vortices (see Figure $8(b)$ and $19(b)$ ). Although the vortex shedding is the main source of force fluctuation, the initial shedding in the far downstream region weakly affects the spanwise variation of the fluctuating pressure on the cylinder surfaces, and hence a good correlation is obtained. However, we find that the correlation pattern does not monotonically vary with $R^{+}$. The forces are the most spanwise uncorrelated for the $R^{+}=0.250$ and $R^{+}=0.375$ cylinders, but improve significantly for the circular cylin$\operatorname{der}\left(R^{+}=0.500\right)$ although the formation length is smaller. We believe this improved correlation is due to the delayed separation angle for the circular cylinder, which in fact has the largest separation angle shown in Table 3. The boundary layer flow before separation is steady and more spanwise uniform than the complex wake flow; the delayed separation narrows the region where the surface pressure can be affected by the wake flow, thus results in a good correlation. It is noted here that the correlation coefficient of the sectional lift $\left(\gamma_{L}\right)$ is high compared with others on high-Re flow past a cylinder 
(e.g., [72]). Since the fluctuating lift is mainly due to the pressure difference on the lat-

620 eral surfaces of the cylinder, this correlation coefficient is determined by the spanwise non-uniformity of the instantaneous local flow field. Referring to Figure 19, it is seen that the separated shear layer on the two lateral surfaces exhibits very good spanwise uniformity for all cylinders at the present Reynolds number, hence the instantaneous pressure difference is well correlated in the spanwise direction, resuting in the high $\gamma_{L}$.

The three-dimensionality of the flow may be quantitatively evaluated by the instantaneous enstrophy. Since the non-uniformity of the flow is primary in the crosssectional plane (significant spanwise vorticity $\omega_{z}$ ) and the spanwise secondary flow is relatively weak, the normalized enstrophy is defined by its primary and secondary components, and the total value as:

$$
\begin{gathered}
E n s_{z}(t)=\frac{1}{\Omega} \int_{\Omega} \omega_{z}^{2}(\mathbf{x}, t) d \Omega \\
E n s_{x y}(t)=\frac{1}{\Omega} \int_{\Omega}\left[\omega_{x}^{2}(\mathbf{x}, t)+\omega_{y}^{2}(\mathbf{x}, t)\right] d \Omega, \\
E n s_{t o t}(t)=E n s_{z}(t)+E n s_{x y}(t),
\end{gathered}
$$

where $\omega$ is the vorticity and $\Omega$ is the whole computational domain, and all three quantities are normalized by $U_{0}^{2} / D^{2}$. Since the enstrophy is vanishingly small far from the cylinder and the definitions in Eqs.(15)-(17) are volume-averaged, the magnitude of the enstrophy depends on the size of the computational domain and our quantifications are only indicative of the comparative difference of the intensity of the flow threedimensionality. The time history of the enstrophy variations are presented in Figure 21 and their time-averaged values are listed in Table 5. The flow past the square cylinder develops strong three-dimensional structures as a result of the early flow separation 
and the evident flow fluctuations (see Figures $8(a)$ and 14(a1-a2)). Both the primary and secondary enstrophy components are noticeably larger than those of the rounded cylinders, with the time-averaged value $\overline{E n s_{x y}}$ about two times that of the rounded cylinders and $\overline{E n s_{z}}$ about $60 \%$ higher. The magnitude of the enstrophy components only slightly decreases with $R^{+}$for the rounded cylinders. For the fraction of primary to total enstrophy, both the instantaneous and time-averaged values of the square cylinder case are around $E n s_{z} / E n s_{t o t}=0.40$ and are smallest among all cases, reflecting the full development of the three-dimensional flow. On the contrary, the flow past the rounded cylinders mainly develops strong spanwise vortices with comparably weaker streamwise and transverse vorticity components, thus the flow exhibits smaller threedimensionality compared with the square cylinder case and the fraction is the smallest for the circular cylinder.

\section{Conclusions}

Direct numerical simulations are performed for flow past cylinders with corners rounded at different radii. The incoming flow is assumed laminar at $R e=1,000$ and flow separation and transition to turbulent occurs. All the corners of the square cylinder are rounded with normalized radius at $R^{+}=0.000$ (square cylinder with sharp corners), $0.125,0,250,0.375$ and 0.500 (circular cylinder). The simulations are conducted using a fourth-order finite difference code. The characteristic quantities produced by the present simulations agree well with those in the literature, and the sufficiency of the spatial resolution and simulation duration is confirmed through careful inspection.

The objective of the present work is to investigate the effect of the corner radius on 

flow patterns, turbulent statistics and three-dimensionality. The rounding of the corners greatly reduces the time-averaged drag as well as the force fluctuations, and the Strouhal number monotonically increases with $R^{+}$. The time-averaged wake recirculation bubble grows significantly in the streamwise direction as the corners are rounded from $R^{+}=0.000$ to $R^{+}=0.125$, while it gradually gets smaller as $R^{+}$further increases. Consequently, the wake flow of the square cylinder recovers the most rapidly in the near-wake but the slowest in the far-wake, forming a much wider wake than other cylinders.

The time-dependent flow patterns are influenced by the corner radius in terms of the flow fluctuation and the quasi-periodic evolution of the shed vortices. Both the coherent and incoherent normal Reynolds stresses $\left(\overline{u^{*} u^{*}} / U_{0}^{2}\right.$ and $\left.\overline{u^{\prime} u^{\prime}} / U_{0}^{2}\right)$ are the most pronounced in the free shear layer and especially in the near-wake close to the reattachment point, and these notably decrease by rounding the corners from $R^{+}=0.000$ to $R^{+}=0.125$ but do not vary much at $R^{+}=0.250$ and 0.375 . The flow is not well organized in solely a time-periodic sense, since the incoherent stress due to turbulent fluctuations and low-frequency modulation is stronger than the coherent one, with the imperfect periodicity mainly observed within the wake recirculation bubble. The flow is most regularized for the circular cylinder as its fluctuation is primarily governed by the coherent evolution and much better time-periodicity is observed in the wake flow. The shed vortices move outwards away from the wake centerline as they convect downstream; the trajectory of the vortex center is roughly the same for the rounded cylinders but is further away from the wake centerline for the square cylinder. The downstream 
convection velocity of the shedding vortex is around $0.8 U_{0}$, which is only slightly affected by $R^{+}$and is consistent with those of the high-Re flows found in the literature. The flow past the square cylinder is strongly three-dimensional in that both the primary and secondary enstrophy are larger than the rounded cylinders. The laminar-turbulent transition occurs the earliest for the square cylinder case, reflecting the least stable separated shear layer flow.

It is noticed based on the above analysis that the multiple quantities related to the

flow patterns may not be monotonically varying with the corner radius $R^{+}$. There could be a drastic variation of the flow pattern between the square cylinder and the partially rounded cylinder cases, and between the latter and the circular cylinder case. The prediction on the variation of a given quantity with respect to the corner radius is not straightforward for such a flow with massive separation.

\section{Acknowledgment}

The authors appreciate the valuable comments from the reviewers. The work was supported by the KAUST Office of Competitive Research Funds under Award No. URF/1/1394-01. The IBM Blue Gene/P Shaheen at KAUST was utilized for the simu-

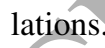

[1] C. H. K. Williamson, Vortex dynamics in the cylinder wake, Annu. Rev. Fluid Mech. 28 (1996) 477-539. doi:10.1146/annurev.fl.28.010196.002401. 
[2] D. J. Tritton, Experiments on the flow past a circular cylinder at low Reynolds numbers, J. Fluid Mech. 6 (1959) 547-567. doi:10.1017/ S0022112059000829.

[3] M. S. Bloor, The transition to turbulence in the wake of a circular cylinder, J. Fluid Mech. 19 (1964) 290-304. doi:10.1017/S0022112064000726.

[4] C. H. K. Williamson, The existence of two stages in the transition to threedimensionality of a cylinder wake, Phys. Fluids 31 (1988) 3165-3168. doi: $10.1063 / 1.866925$.

[5] C. H. K. Williamson, Oblique and parallel modes of vortex shedding in the wake of a circular cylinder at low Reynolds numbers, J. Fluid Mech. 206 (1989) 579627. doi: $10.1017 / \mathrm{S} 0022112089002429$.

[6] R. Mittal, S. Balachandar, Generation of streamwise vortical structures in bluff body wakes, Phys, Rev. Lett. 75 (1995) 1300. doi:10.1103/PhysRevLett. 75.1300.

[7] C. H. K. Williamson, Three-dimensional wake transition, J. Fluid Mech. 328 (1996) 345-407. doi : 10.1017/S0022112096008750.

[8] A. Sohankar, C. Norberg, L. Davidson, Numerical simulation of unsteady lowReynolds number flow around rectangular cylinders at incidence, J. Wind Eng. Ind. Aerod. 69 (1997) 189-201. doi:10.1016/S0167-6105(97) 00154-2.

[9] A. Sohankar, C. Norberg, L. Davidson, Low-Reynolds-number flow around a square cylinder at incidence: study of blockage, onset of vortex shedding and 
outlet boundary condition, Int. J. Numer. Methods Fl. 26 (1998) 39-56. doi : 10 . 1002/(SICI) 1097-0363(19980115) $26: 1<39:$ :AID-FLD623>3. 0 . CO;2-P.

[10] D. H. Yoon, K. S. Yang, C. B. Choi, Flow past a square cylinder with an angle of incidence, Phys. Fluids 22 (2010) 043603. doi : 10.1063/1.3388857.

[11] S.-J. Baek, S. B. Lee, H. J. Sung, Response of a circular cylinder wake to superharmonic excitation, J. Fluid Mech. 442 (2001) 67-88. doi:10.1017/ S0022112001004980.

[12] K. S. Yang, J. Y. Hwang, K. Bremhorst, S. Nesic, Turbulent flow around a rotating stepped cylinder, Phys. Fluids 14 (2002) 1544-1547. doi : 10.1063/1.1455625.

[13] S. Mittal, B. Kumar, Flow past a rotating cylinder, J. Fluid Mech. 476 (2003) 303-334. doi:10.1017/S0022112002002938.

[14] G. V. Papaioannou, D. K. P. Yue, M. S. Triantafyllou, G. E. Karniadakis, Threedimensionality effects in flow around two tandem cylinders, J. Fluid Mech. 558 (2006) 387-413. doi:10.1017/S0022112006000139.

[15] S. R. Kumar, A. Sharma, A. Agrawal, Simulation of flow around a row of square cylinders, J. Fluid Mech. 606 (2008) 369-397. doi:10.1017/ S0022112008001924.

[16] C. L. Liang, G. Papadakis, X. Y. Luo, Effect of tube spacing on the vortex shedding characteristics of laminar flow past an inline tube array: a numerical study, Comput. Fluids 38 (2009) 950-964. doi:10.1016/j.compfluid.2008.10. 005. 
[17] B. S. Carmo, J. R. Meneghini, S. J. Sherwin, Possible states in the flow around two circular cylinders in tandem with separations in the vicinity of the drag inversion spacing, Phys. Fluids 22 (2010) 054101. doi : 10.1063/1.3420111.

[18] M. M. Alam, Y. Zhou, X. W. Wang, The wake of two side-by-side square cylinders, J. Fluid Mech. 669 (2011) 432-471. doi : 10.1017/S0022112010005288.

[19] D. F. L. Labbé, P. A. Wilson, A numerical investigation of the effects of the spanwise length on the 3-D wake of a circular cylinder, J. Fluid Struct. 23 (2007) 1168-1188. doi:10.1016/j.jfluidstructs.2007.05.005.

[20] A. Sohankar, C. Norberg, L. Davidson, Simulation of three-dimensional flow around a square cylinder at moderate Reynolds numbers, Phys. Fluids 11 (1999) 288-306. doi:10.1063/1.869879.

[21] G. L. Larose, A. D’Auteuil, Experiments on 2D rectangular prisms at high Reynolds numbers in a pressurised wind tunnel, J. Wind Eng. Ind. Aerod. 96 (2008) 923-933. doi:10.1016/j.jweia.2007.06.018.

[22] T. Tamura, T. Miyagi, T. Kitagishi, Numerical prediction of unsteady pressures on a square cylinder with various corner shapes, J. Wind Eng. Ind. Aerod. 74 (1998) 531-542. doi:10.1016/S0167-6105(98)00048-8.

[23] T. Tamura, T. Miyagi, The effect of turbulence on aerodynamic forces on a square cylinder with various corner shapes, J. Wind Eng. Ind. Aerod. 83 (1999) 135-145. doi:10.1016/S0167-6105(99)00067-7.

[24] Y. Ueda, M. Kurata, T. Kida, M. Iguchi, Visualization of flow past a square prism 
with cut-corners at the front-edge, J. Visual.-Japan 12 (2009) 383-391. doi: 10.1007/BF03181881.

[25] G. S. He, N. Li, J. J. Wang, Drag reduction of square cylinders with cutcorners at the front edges, Exp. Fluids 55 (2014) 1-11. doi:10.1007/ s00348-014-1745-1.

[26] L. Carassale, A. Freda, M. Marrè-Brunenghi, Experimental investigation on the aerodynamic behavior of square cylinders with rounded corners, J. Fluid Struct. 44 (2014) 195-204. doi:10.1016/j.jfluidstructs.2013.10.010.

[27] J. C. Hu, Y. Zhou, C. Dalton, Effects of the corner radius on the near wake of a square prism, Exp. Fluids 40 (2006) 106-118. doi:10.1007/ s00348-005-0052-2.

[28] J. C. Hu, Y. Zhou, Aerodynamic characteristics of asymmetric bluff bodies, J. Fluid Eng. 131 (2009) 011206. doi:10.1115/1.2979229.

[29] W. Zhang, W. Cheng, W. Gao, A. Qamar, R. Samtaney, Geometrical effects on the airfoil flow separation and transition, Comput. Fluids 116 (2015) 60-73. doi : $10.1016 / j \cdot$ compfluid.2015.04.014.

[30] W. Zhang, R. Samtaney, A direct numerical simulation investigation of the synthetic jet frequency effects on separation control of low-Re flow past an airfoil, Phys. Fluids 27 (2015) 055101. doi:10.1063/1.4919599.

780 [31] W. Zhang, R. Samtaney, Assessment of spanwise domain size effect on the tran- 
sitional flow past an airfoil, Comput. Fluids 124 (2016) 39-53. doi:10.1016/ j.compfluid.2015.10.008.

[32] W. Zhang, R. Samtaney, Numerical simulation and global linear stability analysis of low-Re flow past a heated circular cylinder, Int. J. Heat Mass Tran. 98 (2016) 584-595. doi:10.1016/j.ijheatmasstransfer.2016.03.058

[33] W. Zhang, R. Samtaney, BiGlobal linear stability analysis on low-Re flow past an airfoil at high angle of attack, Phys. Fluids 28 (2016) 044105. doi:10.1063/1. 4945005.

[34] Y. Morinishi, T. S. Lund, O. V. Vasilyev, P. Moin, Fully conservative higher order finite difference schemes for incompressible flow, J. Comput. Phys. 143 (1998) 90-124. doi:10.1006/jcph.1998.5962.

[35] Y. Zang, R. L. Street, J. R. Koseff, A non-staggered grid, fractional step method for time-dependent incompressible Navier-Stokes equations in curvilinear coordinates, J. Comput. Phys. 114 (1994) 18-33. doi:10.1006/jcph.1994.1146.

795 [36] S.-Y. Cao, S. Ozono, Y. Tamura, Y.-J. Ge, H. Kikugawa, Numerical simulation of Reynolds number effects on velocity shear flow around a circular cylinder, J. Fluid Struct. 26 (2010) 685-702. doi:10.1016/j · jfluidstructs.2010.03.003.

[37] C. W. Lei, L. Cheng, K. Kavanagh, Spanwise length effects on three-dimensional modelling of flow over a circular cylinder, Comput. Method Appl. M. 190 (2001) 2909-2923. doi : 10.1016/S0045-7825(00)00272-3.

[38] H. Persillon, M. Braza, Physical analysis of the transition to turbulence in the 
wake of a circular cylinder by three-dimensional Navier-Stokes simulation, J. Fluid Mech. 365 (1998) 23-88. doi:10.1017/S0022112098001116.

[39] A. K. Saha, G. Biswas, K. Muralidhar, Three-dimensional study of flow past square cylinder at low Reynolds numbers, Int. J. Heat Fluid Fl. 24 (2003) 54-66. doi : 10.1016/S0142-727X(02)00208-4.

[40] A. Sohankar, Flow over a bluff body from moderate to high Reynolds numbers using large eddy simulation, Comput. Fluids 35 (2006) 1154-1168. doi:10. 1016/j.compfluid.2005.05.007.

[41] E. C. Maskell, A theory of the blockage effects on bluff bodies and stalled wings in a closed wind tunnel, Tech. rep., Aero. Res. Commun. Rep. Memo. 3400 (1963).

[42] F. M. Najjar, S. Balachandar, Low-frequency unsteadiness in the wake of a normal flat plate, J. Fluid Mech. 370 (1998) 101-147. doi:10.1017/ S0022112098002110.

[43] W. C. Reynolds, A. K. M. F. Hussain, The mechanics of an organized wave in turbulent shear flow. Part 3. Theoretical models and comparisons with experiments, J. Fluid Mech. 54 (1972) 263-288. doi : 10.1017/S0022112072000679.

[44] B. Cantwell, D. Coles, An experimental study of entrainment and transport in the turbulent near wake of a circular cylinder, J. Fluid Mech. 136 (1983) 321-374. doi : 10.1017/S0022112083002189.

[45] D. A. Lyn, S. Einav, W. Rodi, J. H. Park, A laser-doppler velocimetry study of 
ensemble-averaged characteristics of the turbulent near wake of a square cylinder, J. Fluid Mech. 304 (1995) 285-319. doi :10.1017/S0022112095004435.

825 [46] M. Hayakawa, F. Hussain, Three-dimensionality of organized structures in plane turbulent wake, J. Fluid Mech. 206 (1989) 375-404. doi:10.1017/ S0022112089002338.

[47] S. Balachandar, R. Mittal, F. M. Najjar, Properties of the mean recirculation region in the wakes of two-dimensional bluff bodies, J. Fluid Mech. 351 (1997) 167-199. doi:10.1017/S0022112097007179.

[48] M. Breuer, N. Jovičić, Separated flow around a flat plate at high incidence: an LES investigation, J. Turbul. 2 (2001) 1-15. doi : 10.1088/1468-5248/2/1/ 018.

[49] S. Dutta, K. Muralidhar, P. Panigrahi, Influence of the orientation of a square cylinder on the wake properties, Exp. Fluids 34 (2003) 16-23. doi:10.1007/ s00348-002-0484-x.

[50] C. Norberg, Flow around rectangular cylinders: pressure forces and wake frequencies, J. Wind Eng. Ind. Aerod. 49 (1993) 187-196. doi:10.1016/ 0167-6105(93) 90014-F.

[51] A. Okajima, Strouhal numbers of rectangular cylinders, J. Fluid Mech. 123 (1982) 379-398. doi:10.1017/S0022112082003115.

[52] A. Okajima, H. Ueno, H. Sakai, Numerical simulation of laminar and turbulent 
flows around rectangular cylinders, Int. J. Numer. Methods Fl. 15 (1992) 9991012. doi:10.1002/fld.1650150906.

845 [53] A. Sohankar, A LES study of the flow interference between tandem square cylinder pairs, Theor. Comp. Fluid Dyn. 28 (2014) 531-548. doi:10.1007/ s00162-014-0329-2.

[54] C. Evangelinos, G. E. Karniadakis, Dynamics and flow structures in the turbulent wake of rigid and flexible cylinders subject to vortex-induced vibrations, J. Fluid Mech. 400 (1999) 91-124. doi:10.1017/S0022112099006606.

[55] R. D. Henderson, G. E. Karniadakis, Unstructured spectral element methods for simulation of turbulent flows, J. Comput. Phys. 122 (1995) 191-217. doi: 10. 1006/jcph. 1995.1208.

[56] C. Norberg, An experimental investigation of the flow around a circular cylinder: influence of aspect ratio, J. Fluid Mech. 258 (1994) 287-316. doi:10.1017/ S0022112094003332.

[57] C. Norberg, Fluctuating lift on a circular cylinder: review and new measurements, J. Flùid Struct. 17 (2003) 57-96. doi : 10.1016/S0889-9746 (02) 00099-3.

[58] V. D. Narasimhamurthy, H. I. Andersson, Numerical simulation of the turbulent wake behind a normal flat plate, Int. J. Heat Fluid Fl. 30 (2009) 1037-1043. doi : 10.1016/j.ijheatfluidflow.2009.09.002.

[59] H. A. Khaledi, H. I. Andersson, Direct numerical simulation of turbulent flow past a T-beam, J. Turbul. 12 (2011) 1-14. doi:10.1080/14685248.2011.570764. 
[60] F. X. Trias, A. Gorobets, A. Oliva, Turbulent flow around a square cylinder at Reynolds number 22,000: A DNS study, Comput. Fluids 123 (2015) 87-98. doi : 10.1016/j.compfluid.2015.09.013.

[61] P. W. Bearman, Investigation of the flow behind a two-dimensional model with a blunt trailing edge and fitted with splitter plates, J. Fluid Mech. 21 (1965) 241 255. doi:10.1017/S0022112065000162.

[62] A. G. Kravchenko, P. Moin, Numerical studies of flow over a circular cylinder at $\operatorname{Re}_{D}=3900$, Phys. Fluids 12 (2000) 403-417. doi:10.1063/1.870318.

[63] L. Ong, J. Wallace, The velocity field of the turbulent very near wake of a circular cylinder, Exp. Fluids 20 (1996) 441-453. doi:10.1007/BF00189383.

[64] S. Tanaka, S. Murata, An investigation of the wake structures of a circular cylinder using a computer aided flow visualization: 1st Report, generation and dissipation of the vorticities, B. JSME 29 (1986) 1446-1454. doi:/10.1299/jsme1958. 29.1446.

[65] Y. Zhou, R. A. Antonia, Convection velocity measurements in a cylinder wake, Exp. Fluids 13 (1992) 63-70. doi:10.1007/BF00208077.

[66] R. Volino, L. Hultgren, Measurements in separated and transitional boundary layers under low-pressure turbine airfoil conditions, J. Turbomach. 123 (2001) 189197. doi:10.1115/1.1350408.

[67] Y. Zhou, Z. Wang, Effects of surface roughness on separated and transitional flows over a wing, AIAA J. 50 (2012) 593-609. doi:10.2514/1. J051237. 
[68] H. Hu, Z. Yang, An experimental study of the laminar flow separation on a lowReynolds-number airfoil, J. Fluids Eng. 130 (2008) 051101. doi:10.1115/1. 2907416.

[69] N. A. Buchmann, C. Atkinson, J. Soria, Influence of ZNMF jet flow control on the spatio-temporal flow structure over a NACA-0015 airfoil, Exp. Fluids 54 (2013) 1-14. doi:10.1007/s00348-013-1485-7.

[70] J. Jeong, F. Hussain, On the identification of a vortex, J. Fluid Mech. 285 (1995) 69-94. doi:10.1017/S0022112095000462.

[71] L. E. Jones, R. D. Sandberg, N. D. Sandham, Direct numerical simulations of forced and unforced separation bubbles on an airfoil at incidence, J. Fluid Mech. 602 (2008) 175-207. doi:10.1017/S0022112008000864.

[72] P. W. Bearman, E. D. Obasaju, An experimental study of pressure fluctuations on fixed and oscillating square-section cylinders, J. Fluid Mech. 119 (1982) 297321. doi : $10.1017 /$ S0022112082001360. 